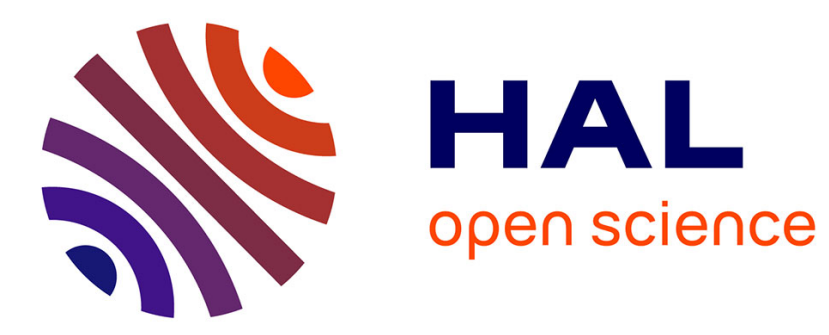

\title{
Homogenization of an elastodynamics system with a strong magnetic field and soft inclusions inducing a viscoelastic effective behavior
}

\author{
Marc Briane, Juan Casado-Diaz
}

\section{- To cite this version:}

Marc Briane, Juan Casado-Diaz. Homogenization of an elastodynamics system with a strong magnetic field and soft inclusions inducing a viscoelastic effective behavior. Journal of Mathematical Analysis and Applications, 2020, 492 (2), pp.124472. 10.1016/j.jmaa.2020.124472 . hal-02303300v2

\section{HAL Id: hal-02303300 \\ https://hal.science/hal-02303300v2}

Submitted on 3 Sep 2020

HAL is a multi-disciplinary open access archive for the deposit and dissemination of scientific research documents, whether they are published or not. The documents may come from teaching and research institutions in France or abroad, or from public or private research centers.
L'archive ouverte pluridisciplinaire HAL, est destinée au dépôt et à la diffusion de documents scientifiques de niveau recherche, publiés ou non, émanant des établissements d'enseignement et de recherche français ou étrangers, des laboratoires publics ou privés. 


\title{
Homogenization of an elastodynamic system with a strong magnetic field and soft inclusions inducing a viscoelastic effective behavior
}

\author{
Marc BRIANE \\ Institut de Recherche Mathématique de Rennes \\ Univ Rennes, INSA de Rennes, CNRS, IRMAR - UMR 6625 \\ mbriane@insa-rennes.fr
}

\author{
Juan CASADO-DÍAZ \\ Dpto. de Ecuaciones Diferenciales y Análisis Numérico \\ Universidad de Sevilla \\ jcasadod@us.es
}

July 23, 2020

\begin{abstract}
In this paper we study homogenization of a linear system of elastodynamics in an elastic body with soft inclusions, which is embedded in a highly oscillating magnetic field. We show two limit behaviors according to the magnetic field. On the one hand, if the magnetic field has at least two different directions at the interface between the hard phase and the soft phase, then the limit of the displacement in the hard phase is independent of time, so that the magnetic field induces an infinite effective mass. On the other hand, if the magnetic field has a constant direction $\xi$ at the interface, then the limit of the displacement in the hard phase involves an additional displacement in the direction $\xi$ which is solution to an elastodynamics equation with a memory mass, a memory stress tensor and memory external forces depending on the initial conditions, which read as time convolutions with some kernel. When the magnetic field has the same direction $\xi$ in the soft phase with smooth inclusions, we prove that the space-average of the kernel is regular and that the limit of the overall displacement in the direction $\xi$ is solution to a viscoelasticity equation.
\end{abstract}

Keywords: elastodynamics, magnetic field, soft inclusions, homogenization, viscoelasticity

AMS subject classification: 74Q10, 74Q15, 35B27, 35L05

\section{Introduction}

This paper is devoted to the asymptotic behavior as $\varepsilon \rightarrow 0$ of the following elastodynamic system posed in a bounded cylinder $Q_{T}=(0, T) \times \Omega$ of $\mathbb{R} \times \mathbb{R}^{3}$,

$$
\begin{cases}\partial_{t t}^{2} u_{\varepsilon}-\operatorname{Div}\left(\mathbf{A}_{\varepsilon}\left(\frac{x}{\varepsilon}\right) \mathbf{e}\left(u_{\varepsilon}\right)\right)+\frac{1}{\varepsilon} b\left(\frac{x}{\varepsilon}\right) \times \partial_{t} u_{\varepsilon}=f & \text { in } Q_{T} \\ u_{\varepsilon}=0 & \text { on }(0, T) \times \partial \Omega \\ u_{\varepsilon}(0, \cdot)=u^{0}, \partial_{t} u_{\varepsilon}(0, \cdot)=v^{0} & \text { in } \Omega,\end{cases}
$$

where the symmetric tensor-valued function $\mathbf{A}_{\varepsilon}$ takes periodically some value $\mathbf{A}_{1}$ in the hard material $\Omega_{\varepsilon, 1}$ and the value $\varepsilon^{2} \mathbf{A}_{2}$ in the soft material $\Omega_{\varepsilon, 2}$, and $b$ is a periodic vector-valued function 
representing a magnetic field which induces the highly oscillating Lorentz force $1 / \varepsilon b(x / \varepsilon) \times \partial_{t} u_{\varepsilon}$. The system of elastodynamics (1.1) is inspired by a coupling magneto-elastodynamics model of [2, Section 9.3]. Here, assuming that the elastic body is a poor conductor and that the electric Lorentz force is negligible against the magnetic Lorentz force, the coupling leads us to the simpler elastodynamics equation (1.1) with the magnetic Lorentz force.

Homogenization of wave equations with varying coefficients was first studied by Colombini, Spagnolo [7], and extended by Francfort, Murat [8]. In these works, roughly speaking the varying matrix-rigidity of the material is assumed to be uniformly bounded, coercive and does not oscillate in time which leads us to a limit wave equation of the same nature. However, when the above uniformity conditions are not satisfied or when the rigidity coefficients oscillate in time, the nature of the equation is not in general preserved. On the one hand, in the case of an elastodynamics system with soft inclusions Ávila et al. [3] have highlighted the appearance at a fixed frequency of an effective negative mass related to the existence of phononic band gaps. In the stationary elasticity case with soft inclusions Zhikov and Pasthukova [18] have shown the existence of gaps in the continuous spectrum of the homogenized operator. Otherwise, Smyshlyaev [16] (see also [9] for some extension) has studied elastic waves in highly anisotropic periodic composites. More generally, observing that high-contrast composite materials (mixing soft and hard phases) may induce an anisotropic mass at a fixed frequency, Milton, Willis [12] have proposed a modification of Newton's second law in which the relation between the force and the acceleration is non-local in time. On the other hand, a nonlocal term was obtained in [6] for a wave equation with a first order term with periodic coefficients in space and time. More recently, in the absence of soft inclusions, i.e. $\mathbf{A}_{\varepsilon}=\mathbf{A}_{1}$, the present authors [5] have obtained for system (1.1) but in a non-periodic framework a homogenized system involving both an increase of the effective mass and a nonlocal term due to a time-oscillating Lorentz force. In this work, the increase of mass is due to a highly space-oscillating magnetic field in the spirit of homogenization of the hydrodynamic problem studied by Tartar [17]. Moreover, the presence in [5] of a time-oscillating magnetic field induces a non-local term in the homogenized system.

In the present work, we consider both a highly space-oscillating magnetic field and soft inclusions. Moreover, contrary to [3] and [12] rather than fixing the frequency we study homogenization of the non-stationary elastodynamic system (1.1). We obtain two asymptotic behaviors for system (1.1) (see Theorem 2.2) according to the following alternative:

- If the magnetic field has two or more different directions at the interface between the soft and the hard material, then the displacement in the hard phase $\chi_{\Omega_{\varepsilon, 1}} u_{\varepsilon}$ weakly converges in $L^{2}\left(Q_{T}\right)^{3}$ to the stationary function $\left|Y_{1}\right| u^{0}$, where $Y_{1}$ is the cell period of the hard phase. From the point of view of the hard phase the strong magnetic field thus induces an isotropic infinite mass which blocks the displacement.

- If the magnetic field has a fixed direction $\xi$ at the interface between the soft and the hard material, then the displacement $\chi_{\Omega_{\varepsilon, 1}} u_{\varepsilon}$ weakly converges to $\left|Y_{1}\right|\left(u^{0}+\alpha \xi\right)$ in $L^{2}\left(Q_{T}\right)^{3}$, where the scalar function $\alpha$ is solution to an equation of elastodynamics involving a memory mass, a memory stress tensor and memory external forces depending on the initial displacement $u^{0}$, the initial velocity $v^{0}$ and the force $f$. The memory terms read as time-convolutions with a matrix-valued kernel $\bar{K}$ or its derivative $\partial_{t} \bar{K}$ defined on $(0, T) \times Y_{2}$, where $Y_{2}$ is the cell period of the soft phase. Contrary to the first case, the strong magnetic field induces an anisotropic effective mass (in the spirit of [12]) which is only infinite in the direction perpendicular to the field.

In the second case, assuming that the magnetic field has the same direction $\xi$ in $Y_{2}$ and the tensor $\mathbf{A}_{2}$ is constant (see Example 2.7), it turns out that the function $\alpha$ can be expressed with 
some kernel $L$ as the time convolution

$$
\alpha=L *_{t}(\bar{u} \cdot \xi+G) \text { in } Q_{T}
$$

where $\bar{u}$ is the weak limit of the overall displacement $u_{\varepsilon}$ in $L^{2}\left(Q_{T}\right)^{3}$, and $G$ is a term depending on the initial conditions $u^{0}, v^{0}$ and the external force $f$. Therefore, the homogenized equation satisfied by $\alpha$ can be regarded as a viscoelasticity type equation

$$
\begin{cases}\partial_{t t}(\bar{u} \cdot \xi)-\operatorname{div}_{x} \sigma=f \cdot \xi+\operatorname{div}_{x}\left(\mathbf{A}_{1}^{*} \mathbf{e}_{x}\left(u^{0}\right) \xi\right) & \text { in } Q_{T} \\ (\bar{u} \cdot \xi)(0, \cdot)=u^{0} \cdot \xi & \text { in } \Omega,\end{cases}
$$

satisfied by the overall macroscopic displacement $\bar{u} \cdot \xi$ in the direction $\xi$ and the stress tensor $\sigma$ which are connected by the relation

$$
\sigma:=A_{1}^{*} \nabla_{x}\left(L *_{t}(\bar{u} \cdot \xi+G)\right) \text { in } Q_{T},
$$

for some homogenized elliptic tensor $\mathbf{A}_{1}^{*}$ and a positive definite matrix $A_{1}^{*}$ depending on $\mathbf{A}_{1}^{*}$.

Homogenization of an elastodynamics equation of type (1.1) was studied by Sánchez-Palencia [14, Sect. 4, Chap. 6] (see also [1] for a similar model with time-dependent coefficients) replacing roughly speaking the first-order derivative term $1 / \varepsilon b(x / \varepsilon) \times \partial_{t} u_{\varepsilon}$ by the third-order derivative term $\operatorname{div}\left(\mathbb{B}(x / \varepsilon) \mathbf{e}_{x}\left(\partial_{t} u_{\varepsilon}\right)\right)$, where $\mathbb{B}$ is some periodic tensor-valued function. Therefore, starting from a viscoelastic behavior given by the stress-strain law

$$
\sigma_{\varepsilon}(t, x)=\mathbb{A}(x / \varepsilon) \mathbf{e}_{x}\left(u_{\varepsilon}\right)+\mathbb{B}(x / \varepsilon) \mathbf{e}_{x}\left(\partial_{t} u_{\varepsilon}\right),
$$

Sánchez-Palencia obtained a nonlocal limit viscoelasticity equation with a memory term, which is similar to equation (1.3). However in our context, we start from the first-order time derivative Lorentz force $1 / \varepsilon b(x / \varepsilon) \times \partial_{t} u_{\varepsilon}$ without any a priori viscoelastic behavior, and the limit viscoelasticity equation (1.3) is only induced by the homogenization process thanks to the combination of the strong oscillating magnetic field and the soft inclusions. Such a derivation by homogenization of a viscoelastic behavior from an elastodynamic system is original to best of our knowledge.

The proof of Theorem 2.2 is based on a two-scale convergence result (see Theorem 2.1) in the sense of Nguetseng-Allaire [13, 4]. Here, the main difficulty is to pass to the two-scale limit in the highly oscillating Lorentz force, which needs a suitable matrix-valued test function. Then, we deduce from the variational formulation of the two-scale limit of system (1.1) the homogenized equation in the direction of the magnetic field. This is the most delicate part of the proof which involves some matrix-valued kernel $\bar{K}$ the derivative of which $\partial_{t} \bar{K}$ is a priori only in $L^{\infty}\left(0, T ; L^{2}\left(Y_{2}\right)\right)^{3 \times 3}$. We prove (see Proposition 2.6) that the space-average of $\bar{K}$ belongs to $W^{1, \infty}(0, T)^{3 \times 3}$ assuming that the magnetic field $b$ has a constant direction in $Y_{2}$, the tensor $\mathbf{A}_{2}$ is constant in $Y_{2}$ and $Y_{2}$ has a smooth boundary. This additional regularity of the kernel allows us to derive the limit viscoelasticity equation (1.3).

\section{Notation}

- $Y$ denotes the unit cube $(0,1)^{3}$ of $\mathbb{R}^{3}$.

- $\Omega$ denotes a regular (satisfying at least the interior cone condition) bounded open set of $\mathbb{R}^{3}$, and $Q_{T}$ the cylinder $(0, T) \times \Omega$ for $T>0$.

- $|E|$ denotes the Lebesgue measure of a measurable set $E$ of $\mathbb{R}^{3}$. 
- denotes the scalar product in $\mathbb{R}^{3}$, : denotes the scalar product in $\mathbb{R}^{3 \times 3}$, and $|\cdot|$ denotes the associated norm in both cases.

- $\left(e_{1}, e_{2}, e_{3}\right)$ denotes the canonical basis of $\mathbb{R}^{3}$.

- $\mathbb{R}^{3 \times 3}$ denotes the set of the $(3 \times 3)$ real matrices, and $\mathbb{R}_{s}^{3 \times 3}$ denotes the set of symmetric matrices in $\mathbb{R}^{3 \times 3}$ ).

- $I$ denotes the unit matrix of $\mathbb{R}^{3 \times 3}$.

- For $\xi, \eta \in \mathbb{R}^{3}, \xi \otimes \eta$ denotes the matrix in $\mathbb{R}^{3 \times 3}$ with entries $\xi_{i} \eta_{j}$, and $\xi \odot \eta$ the symmetrized matrix of $\xi \otimes \eta$.

- $\mathscr{L}(E)$ denotes the set of linear mappings from a vector space $E$ into itself.

- A denotes any $Y$-periodic symmetric tensor-valued function in $L^{\infty}\left(Y ; \mathscr{L}\left(\mathbb{R}_{s}^{3 \times 3}\right)\right)$ which is uniformly elliptic, i.e. there exists a constant $a>0$ such that

$$
\mathbf{A}(y) M: M \geq a M: M, \quad \text { a.e. } y \in Y, \forall M \in \mathbb{R}_{s}^{3 \times 3} .
$$

- $\mathbf{e}(u)$ denotes the symmetrized gradient of a vector-valued function $u$.

- Div denotes the vector-valued divergence operator taking the divergence of each row of a matrix-valued function.

- $C_{c}^{\infty}(U)$ denotes the set of smooth functions with compact support in an open set $U$ of $\mathbb{R}^{3}$.

- $L_{\sharp}^{p}(Y)$, resp. $W_{\sharp}^{1, p}(Y)$, denotes the set of the $Y$-periodic functions defined in $\mathbb{R}^{3}$ which belong to $L_{\text {loc }}^{p}\left(\mathbb{R}^{3}\right)$, resp. $W_{\text {loc }}^{1, p}\left(\mathbb{R}^{3}\right)$.

- $\rightarrow$ denotes a strong convergence, $\rightarrow$ a weak convergence, and $\stackrel{2 s}{\rightarrow}$ the two-scale convergence.

- $o_{\varepsilon}(1)$ denotes a sequence of $\varepsilon$ which converges to zero as $\varepsilon \rightarrow 0$, and which may vary from line to line.

- $C$ denotes a positive constant which may vary from line to line.

Recall the definition of the two-scale convergence of Nguetseng-Allaire in the case of an open cylinder $Q_{T}=(0, T) \times \Omega$ of $\mathbb{R} \times \mathbb{R}^{3}$.

Definition $1.1([13,4])$. A bounded sequence $v_{\varepsilon}(t, x)$ in $L^{2}\left(Q_{T}\right)$ is said to two-scale converge to the function $v(t, x, y)$ in $L^{2}\left(Q_{T} ; L_{\sharp}^{2}(Y)\right)$ if

$$
\forall \varphi \in C_{c}^{\infty}\left(Q_{T} ; C_{\sharp}^{\infty}(Y)\right), \quad \lim _{\varepsilon \rightarrow 0} \int_{Q_{T}} v_{\varepsilon}(t, x) \varphi\left(t, x, \frac{x}{\varepsilon}\right) d t d x=\int_{Q_{T} \times Y} v(t, x, y) \varphi(t, x, y) d t d x d y,
$$

where $C_{c}^{\infty}\left(Q_{T} ; C_{\sharp}^{\infty}(Y)\right)$ denotes the set of functions $\varphi(x, y)$ in $C^{\infty}\left(Q_{T} \times \mathbb{R}^{3}\right)$ compactly supported in $x \in Q_{T}$ and $Y$-periodic in $y$. In particular, this implies that

$$
v_{\varepsilon}(t, x) \rightarrow \int_{Y} v(t, x, y) d y \quad \text { in } L^{2}\left(Q_{T}\right)
$$




\section{Statement of the result}

\subsection{Formulation of the problem}

Let $Y$ be the unit cube in $\mathbb{R}^{3}$, let $Y_{2}$ be a smooth open set such that $\overline{Y_{2}} \subset Y$, and such that $Y_{1}:=Y \backslash \overline{Y_{2}}$ is a connected set. Then, for a given regular (satisfying at least the interior cone condition) bounded open set $\Omega$ of $\mathbb{R}^{3}$, define the open sets

$$
\Omega_{\varepsilon, 1}:=\Omega \backslash \bigcup_{k \in \mathbb{Z}^{3}} \varepsilon\left(k+\overline{Y_{2}}\right), \quad \Omega_{\varepsilon, 2}:=\Omega \backslash \overline{\Omega_{\varepsilon, 1}} .
$$

For a given $T>0$, we also define the cylinder

$$
Q_{T}:=(0, T) \times \Omega .
$$

Let $\mathbf{A}_{1} \in L_{\sharp}^{\infty}\left(Y_{1} ; \mathscr{L}\left(\mathbb{R}_{s}^{3 \times 3}\right)\right), \mathbf{A}_{2} \in L_{\sharp}^{\infty}\left(Y_{2} ; \mathscr{L}\left(\mathbb{R}_{s}^{3 \times 3}\right)\right)$ be two uniformly elliptic periodic symmetric tensor-valued functions (see $(1.5)^{\circ}$ ), and $b \in L_{\sharp}^{\infty}(Y)^{3}$ be a $Y$-periodic vector-valued function. Then, for $f \in L^{2}\left(Q_{T}\right)^{3}, u^{0} \in H_{0}^{1}(\Omega)^{3}$ and $v^{0} \in L^{2}(\Omega)^{3}$, we consider the problem of elastodynamics

$$
\left\{\begin{array}{l}
\frac{d^{2}}{d t^{2}} \int_{\Omega} u_{\varepsilon} \cdot v d x+\int_{\Omega_{\varepsilon, 1}} \mathbf{A}_{1}\left(\frac{x}{\varepsilon}\right) \mathbf{e}\left(u_{\varepsilon}\right): \mathbf{e}(v) d x+\varepsilon^{2} \int_{\Omega_{\varepsilon, 2}} \mathbf{A}_{2}\left(\frac{x}{\varepsilon}\right) \mathbf{e}\left(u_{\varepsilon}\right): \mathbf{e}(v) d x \\
\quad+\frac{1}{\varepsilon} \int_{\Omega}\left(b\left(\frac{x}{\varepsilon}\right) \times \partial_{t} u_{\varepsilon}\right) \cdot v d x=\int_{\Omega} f \cdot v d x \text { in } \Omega, \quad \forall v \in H_{0}^{1}(\Omega)^{3} \\
u_{\varepsilon}=0 \text { on }(0, T) \times \partial \Omega \\
u_{\varepsilon}(0, \cdot)=u^{0}, \partial_{t} u_{\varepsilon}(0, \cdot)=v^{0} \text { in } \Omega,
\end{array}\right.
$$

which denoting

$$
\mathbf{A}_{\varepsilon}:=\chi_{Y_{1}} \mathbf{A}_{1}+\varepsilon^{2} \chi_{Y_{2}} \mathbf{A}_{2}
$$

can also be written as

$$
\begin{cases}\partial_{t t}^{2} u_{\varepsilon}-\operatorname{Div}\left(\mathbf{A}_{\varepsilon}\left(\frac{x}{\varepsilon}\right) \mathbf{e}\left(u_{\varepsilon}\right)\right)+\frac{1}{\varepsilon} b\left(\frac{x}{\varepsilon}\right) \times \partial_{t} u_{\varepsilon}=f & \text { in } Q_{T} \\ u_{\varepsilon}=0 & \text { on }(0, T) \times \partial \Omega \\ u_{\varepsilon}(0, \cdot)=u^{0}, \partial_{t} u_{\varepsilon}(0, \cdot)=v^{0} & \text { in } \Omega .\end{cases}
$$

The weak variational formulation (2.1) has a unique solution in $W^{1, \infty}\left(0, T ; L^{2}(\Omega)\right)^{3} \cap L^{\infty}\left(0, T ; H_{0}^{1}(\Omega)\right)^{3}$ (see, e.g., [11, Chapter 3, Section 8]).

\subsection{Statement of the results}

The following result provides a variational problem in terms of the two-scale limits of $u_{\varepsilon}, \partial_{t} u_{\varepsilon}$ and $\mathbf{e}\left(u_{\varepsilon}\right)$.

Theorem 2.1. Assume that the magnetic field b satisfies the equality

$$
\int_{Y_{1}} b d y=0
$$


Then, we have the following two-scale convergences

$$
\left\{\begin{array}{l}
u_{\varepsilon} \stackrel{2 s}{\rightarrow} u_{1}+u_{2} \\
\partial_{t} u_{\varepsilon} \stackrel{2 s}{\longrightarrow} \partial_{t} u_{1}+\partial_{t} u_{2} \\
\chi_{\Omega_{\varepsilon, 1}} \mathbf{e}\left(u_{\varepsilon}\right) \stackrel{2 s}{\rightarrow} \chi_{Y_{1}}\left(\mathbf{e}_{x}\left(u_{1}\right)+\mathbf{e}_{y}\left(u_{3}\right)\right) \\
\chi_{\Omega_{\varepsilon, 2}} \varepsilon \mathbf{e}\left(u_{\varepsilon}\right) \stackrel{2 s}{\rightarrow} \mathbf{e}_{y}\left(u_{2}\right)
\end{array}\right.
$$

where the functions $u_{1}, u_{2}, u_{3}$ satisfy the conditions

$$
\left\{\begin{array}{c}
u_{1} \in W^{1, \infty}\left(0, T ; L^{2}(\Omega)\right)^{3} \cap L^{\infty}\left(0, T ; H_{0}^{1}(\Omega)\right)^{3}, u_{1}(0, \cdot)=u^{0} \text { in } \Omega, \\
u_{2} \in W^{1, \infty}\left(0, T ; L^{2}\left(\Omega ; L^{2}\left(Y_{2}\right)\right)\right)^{3} \cap L^{\infty}\left(0, T ; L^{2}\left(\Omega ; H_{0}^{1}\left(Y_{2}\right)\right)^{3}, u_{2}(0, \cdot, \cdot)=0 \text { in } \Omega \times Y_{2},\right. \\
u_{3} \in L^{\infty}\left(0, T ; L^{2}\left(\Omega ; H_{\sharp}^{1}\left(Y_{1}\right)\right)\right)^{3}, \\
b(y) \times\left(u_{1}(t, x)+u_{2}(t, x, y)\right)=b(y) \times u^{0}(x) \text { a.e. }(t, x, y) \in Q_{T} \times Y_{2} .
\end{array}\right.
$$

The functions $u_{1}, u_{2}, u_{3}$ are the unique solutions satisfying (2.5), up to a rigid displacement $y \mapsto \lambda(t, x)$ for $u_{3}$, to the variational problem

$$
\begin{aligned}
& -\int_{Q_{T} \times Y}\left(\partial_{t} u_{1}+\partial_{t} u_{2}\right) \cdot\left(\partial_{t} \varphi_{1}+\partial_{t} \varphi_{2}\right) d t d x d y-\int_{\Omega \times Y} v^{0} \cdot\left(\varphi_{1}+\varphi_{2}\right)(0, x, y) d x d y \\
& +\int_{Q_{T} \times Y_{1}} \mathbf{A}_{1}\left(\mathbf{e}_{x}\left(u_{1}\right)+\mathbf{e}_{y}\left(u_{3}\right)\right):\left(\mathbf{e}_{x}\left(\varphi_{1}\right)+\mathbf{e}_{y}\left(\varphi_{3}\right)\right) d t d x d y+\int_{Q_{T} \times Y_{2}} \mathbf{A}_{2} \mathbf{e}_{y}\left(u_{2}\right): \mathbf{e}_{y}\left(\varphi_{2}\right) d t d x d y \\
& +\int_{Q_{T} \times Y_{1}}\left(b \times \partial_{t} u_{1}\right) \cdot \varphi_{3} d t d x d y-\int_{Q_{T} \times Y_{1}}\left(b \times u_{3}\right) \cdot \partial_{t} \varphi_{1} d t d x d y \\
& =\int_{Q_{T} \times Y} f \cdot\left(\varphi_{1}+\varphi_{2}\right) d t d x d y,
\end{aligned}
$$

for any functions $\varphi_{1}, \varphi_{2}, \varphi_{3}$ satisfying

$$
\left\{\begin{array}{c}
\varphi_{1} \in W^{1,1}\left(0, T ; L^{2}(\Omega)\right)^{3} \cap L^{1}\left(0, T ; H_{0}^{1}(\Omega)\right)^{3}, \varphi_{1}(T, \cdot)=0 \text { in } \Omega, \\
\varphi_{2} \in W^{1,1}\left(0, T ; L^{2}\left(\Omega \times Y_{2}\right)\right)^{3} \cap L^{1}\left(0, T ; L^{2}\left(\Omega ; H_{0}^{1}\left(Y_{2}\right)\right)\right)^{3}, \varphi_{2}(T, \cdot, \cdot)=0 \text { in } \Omega \times Y_{2}, \\
\varphi_{3} \in L^{1}\left(0, T ; L^{2}\left(\Omega ; H_{\sharp}^{1}\left(Y_{1}\right)\right)\right)^{3}, \\
b(y) \times\left(\varphi_{1}(t, x)+\varphi_{2}(t, x, y)\right)=0 \quad \text { a.e. }(t, x, y) \in Q_{T} \times Y_{2} .
\end{array}\right.
$$

The next result provides a limit equation for the function $u_{1}$ which represents the macroscopic displacement in the hard material 1.

Theorem 2.2. Assume that condition (2.3) holds and that

$$
b \neq 0 \quad \text { a.e. in } Y_{2}, \quad b \in H^{1}\left(Y_{2}\right)^{3} \quad \text { and } \quad \frac{b \otimes b}{|b|^{2}} \in H^{1}\left(Y_{2}\right)^{3 \times 3} .
$$

Then, we have the following alternative:

- If

$$
\operatorname{dim}\left(\operatorname{Span}\left\{b(y): y \in \partial Y_{2}\right\}\right) \geq 2,
$$


then

$$
u_{1}(t, x)=u^{0}(x) \text { a.e. }(t, x) \in Q_{T},
$$

and there exists a matrix-valued kernel $\bar{K}:(0, T) \times Y_{2} \rightarrow \mathbb{R}^{3 \times 3}$ given by (3.22) below, with

$$
\left\{\begin{array}{l}
\bar{K}(t, y)\left(\mathbb{R}^{3}\right) \subset \mathbb{R} b(y) \text { a.e. }(t, y) \in(0, T) \times Y_{2}, \\
\bar{K} \in L^{\infty}\left(0, T ; H_{0}^{1}\left(Y_{2}\right)\right)^{3 \times 3} \cap W^{1, \infty}\left(0, T ; L^{2}\left(Y_{2}\right)\right)^{3 \times 3} \cap W^{2, \infty}\left(0, T ; H^{-1}\left(Y_{2}\right)\right)^{3 \times 3},
\end{array}\right.
$$

such that

$$
u_{2}(t, x, y)=\bar{K}(t, y) v^{0}(x)+\int_{0}^{t} \bar{K}(t-s, y) f(s, x) d s \quad \text { a.e. }(t, x, y) \in Q_{T} \times Y_{2} .
$$

- If $b_{\mid \partial Y_{2}}$ has a fixed direction $\xi$ with $|\xi|=1$, then we have

$$
\begin{aligned}
u_{1}(t, x)-u^{0}(x)=\alpha(t, x) \xi \quad \text { a.e. }(t, x) \in Q_{T}, \\
u_{2}(t, x, y)=\bar{K}(t, y) v^{0}(x)+\int_{0}^{t} \bar{K}(t-s, y) f(s, x) d s-\int_{0}^{t} \partial_{t} \bar{K}(t-s, y) \partial_{s} \alpha(s, x) \xi d s, \\
-\left(I-\frac{b(y) \otimes b(y)}{|b(y)|^{2}}\right) \alpha(t, x) \xi \text { a.e. }(t, x, y) \in Q_{T} \times Y_{2},
\end{aligned}
$$

and the function $\alpha$ is the unique solution in $W^{1, \infty}\left(0, T ; L^{2}(\Omega)\right) \cap L^{\infty}\left(0, T ; H_{0}^{1}(\Omega)\right)$ to the problem

$$
\left\{\begin{array}{l}
\partial_{t t}\left[M^{*} \alpha-\int_{0}^{t} \bar{K}_{1}(t-s) \partial_{s} \alpha(s, x) d s\right]+\lambda^{*} \cdot \nabla_{x}\left(\partial_{t} \alpha\right)-\operatorname{div}_{x}\left(A_{1}^{*} \nabla_{x} \alpha\right) \\
+c^{*} \alpha-\int_{0}^{t}\left(\int_{Y_{2}} \mathbf{A}_{2}\left(\mathbf{e}_{y} \partial_{t} \bar{K}(t-s, y) \xi\right): \mathbf{e}_{y}(\hat{b}) d y\right) \partial_{s} \alpha(s, x) d s=\mu^{*} \cdot f+F \quad \text { in } Q_{T} \\
\alpha(0, \cdot)=0 \quad \text { in } \Omega
\end{array}\right.
$$

where

$$
\begin{gathered}
\hat{b}(y):=\frac{b(y) \otimes b(y)}{|b(y)|^{2}} \xi, \quad \text { for } y \in Y_{2}, \\
\bar{K}_{1}(t):=\int_{Y_{2}} \partial_{t} \bar{K}(t, y):(\xi \odot \xi) d y, \quad \text { for } t \in(0, T),
\end{gathered}
$$

$F$ is the memory force term acting on the initial displacement $u^{0}$, the initial velocity $v^{0}$ and the original force $f$ given by

$$
\begin{aligned}
& F(t, x):=-\partial_{t t}\left[\int_{Y_{2}} \bar{K}(t, y):\left(\xi \otimes v^{0}(x)\right) d y\right]-\int_{Y_{2}} \mathbf{A}_{2} \mathbf{e}_{y}\left(\bar{K}(t, y) v^{0}(x)\right): \mathbf{e}_{y}(\hat{b}) d y \\
& -\partial_{t t}\left[\int_{Y_{2}}\left(\int_{0}^{t} \bar{K}(t-s, y) f(s, x) d s\right) \cdot \xi d y\right] \\
& -\int_{Y_{2}} \mathbf{A}_{2} \mathbf{e}_{y}\left(\int_{0}^{t} \bar{K}(t-s, y) f(s, x) d s\right): \mathbf{e}_{y}(\hat{b}) d y+\operatorname{div}_{x}\left(\mathbf{A}_{1}^{*} \mathbf{e}\left(u^{0}\right) \xi\right),
\end{aligned}
$$

and $M^{*}, c^{*}>0, \lambda^{*}, \mu^{*} \in \mathbb{R}^{3}, \mathbf{A}_{1}^{*} \in \mathscr{L}\left(\mathbb{R}_{s}^{3 \times 3}\right)$ which is elliptic, $A_{1}^{*} \in \mathbb{R}_{s}^{3 \times 3}$ which is positive definite, are the homogenized quantities defined by (3.31) and (3.32) below. 
Theorem 2.1 and Theorem 2.2 are proved in Section 3.

As a consequence of Theorem 2.1 and Theorem 2.2 we get the weak limits of the displacement $u_{\varepsilon}$ in each material.

\section{Corollary 2.3.}

- If (2.9) is satisfied, we have

$$
\begin{cases}\chi_{\Omega_{\varepsilon, 1}} u_{\varepsilon} \rightarrow\left|Y_{1}\right| u_{0}(x) & L^{2}\left(Q_{T}\right)^{3} \\ \chi_{\Omega_{\varepsilon, 2}} u_{\varepsilon} \rightarrow\left|Y_{2}\right| u_{0}(x)+\int_{Y_{2}} u_{2}(t, x, y) d y & L^{2}\left(Q_{T}\right)^{3},\end{cases}
$$

where $u_{2}$ is given by (2.12).

- Otherwise, we have

$$
\begin{cases}\chi_{\Omega_{\varepsilon, 1}} u_{\varepsilon} \rightarrow\left|Y_{1}\right|\left(u_{0}(x)+\alpha(t, x) \xi\right) & L^{2}\left(Q_{T}\right)^{3} \\ \chi_{\Omega_{\varepsilon, 2}} u_{\varepsilon} \rightarrow\left|Y_{2}\right|\left(u_{0}(x)+\alpha(t, x) \xi\right)+\int_{Y_{2}} u_{2}(t, x, y) d y & L^{2}\left(Q_{T}\right)^{3}\end{cases}
$$

where $\alpha$ is the solution to problem (2.15) and $u_{2}$ is given by (2.14).

Remark 2.4. The strong magnetic field $b$ induces an effective mass which is:

- Infinite when $b$ has at least two directions at the interface between the two materials. In this case the macroscopic displacement $u_{1}$ in material 1 remains equal to the initial displacement $u^{0}$.

- Infinite in the vector space $\xi^{\perp}$ when $b$ has a fixed direction $\xi$ at the interface between the two materials. In this case, the macroscopic displacement $u_{1}$ is solution to the homogenized equation (2.15) in the direction $\xi$ involving, through the kernel $\bar{K}$, a memory mass, a memory stress tensor, and memory external forces depending both on the initial velocity $v^{0}$ and the original force $f$.

On the one hand, in the absence of magnetic field and for a fixed frequency Ávila et al. [3] showed the possible appearance of a negative mass related to phononic band gaps due to similar soft inclusions in the elastic matrix. On the other hand, in the absence of soft inclusions the authors [5] showed the increase of mass due to the magnetic field. Here, the simultaneous presence of a strong magnetic field and soft inclusions leads us to an elastodynamics equation in the direction of the magnetic field involving various memory effects. In the Example 2.7 below we study a simpler case where the limit equation reads as a kind of viscoelasticity equation in the direction of the magnetic field.

Remark 2.5. When $b$ has a fixed direction $\xi$ at the interface between the two materials, by (2.11) and (2.17) the kernel $\bar{K}_{1}$ is in $L^{\infty}(0, T)^{3 \times 3}$. If moreover $\bar{K}_{1}$ belongs to $W^{1,1}(0, T)^{3 \times 3}$, then integrating by parts we get that

$$
\int_{0}^{t} \bar{K}_{1}(t-s) \partial_{s} \alpha(s, x) d s=\bar{K}_{1}(0) \alpha(t, x)+\int_{0}^{t} \partial_{t} \bar{K}_{1}(t-s) \alpha(s, x) d s .
$$

Therefore, the entry in square brackets of (2.15)

$$
\left(M^{*}-\bar{K}_{1}(0)\right) \alpha(t, x)-\int_{0}^{t} \partial_{t} \bar{K}_{1}(t-s) \alpha(s, x) d s
$$


can be regarded as a product mass $\times$ displacement in the direction $\xi$, where the effective mass is the difference of the isotropic constant mass $M^{*}-\bar{K}_{1}(0)$ and the memory mass induced by the kernel $\partial_{t} \bar{K}_{1}$. If we only consider the constant mass in (2.21), then the formula (3.32) for $M^{*}$ yields

$$
M^{*}-\bar{K}_{1}(0)=\left|Y_{1}\right|+m^{*}+\int_{Y_{2}}|\hat{b}|^{2} d y-\bar{K}_{1}(0) .
$$

On the other hand, using the expression (2.17) for $\bar{K}_{1}$, computing the derivative of the series expansion (3.22) of $\bar{K}$ and taking into account the definition (3.20) of $h_{j}$ and $\bar{h}_{j}$, we get

$$
\begin{aligned}
& \bar{K}_{1}(0)=\sum_{i=1}^{\infty} \int_{Y_{2}}\left(h_{i}(y) \otimes \bar{h}_{i}\right):(\xi \otimes \xi) d y \\
& =\sum_{i=1}^{\infty}\left|\int_{Y_{2}} h_{i} \cdot \xi d y\right|^{2}=\sum_{i=1}^{\infty}\left|\int_{Y_{2}} h_{i} \cdot \hat{b} d y\right|^{2}=\int_{Y_{2}}|\hat{b}|^{2} d y .
\end{aligned}
$$

Thus, we have

$$
M^{*}-\bar{K}_{1}(0)=\left|Y_{1}\right|+m^{*},
$$

where by (3.31) $m^{*} \geq 0$. Actually, we may have $m^{*}=0$ (see Example 2.7 below) so that

$$
0<M^{*}-\bar{K}_{1}(0)=\left|Y_{1}\right|<1=\text { the initial mass in equation (2.2). }
$$

In this case we obtain an apparent decrease of the effective mass contrary to the increase of mass in [5] in the absence of soft inclusions. However, the presence of soft inclusions in [3] may induce an arbitrary (possibly negative) mass in some regime but at a fixed frequency. Therefore, a definition of the effective mass in the limit equation (2.15) seems delicate to specify due to the memory term in (2.21). In the particular situation of Example 2.7 below we will give another interpretation of this memory term.

The following result gives a particular case where Remark 2.5 applies.

Proposition 2.6. Assume that the vector-valued tensor $\mathbf{A}_{2}$ is constant in $Y_{2}$, the vector-valued function $b$ has a constant direction $\xi$ in $Y_{2}$, i.e. $\hat{b}=\xi$ in $Y_{2}$, and $Y_{2}$ has a $C^{2}$ boundary. Then, the kernel $\bar{K}_{1}$ is in $W^{1, \infty}(0, T)$.

The proof of Proposition 2.6 is given in Section 3.

Example 2.7. Consider a particular case where there exists a unit vector $\xi \in \mathbb{R}^{3}$ and a scalar function $\gamma \in H_{\sharp}^{1}(Y)$ such that

$$
b(y)=\gamma(y) \xi \text { a.e. } y \in Y, \quad \int_{Y_{1}} \gamma(y) d y=0, \quad \gamma(y) \neq 0 \text { a.e. } y \in Y_{2} \text {. }
$$

From formulas (3.26), (3.31) and (3.32) below we can deduce that

$$
M^{*}=1, \quad c^{*}=0, \quad \lambda^{*}=0, \quad \mu^{*}=\xi .
$$

Then, by the two-scale convergence (2.4) combined with (2.13) and (2.14) the weak limit $\bar{u}$ of $u_{\varepsilon}$ in $L^{2}\left(Q_{T}\right)^{3}$ is given by

$$
\begin{aligned}
\bar{u}(t, x)= & u^{0}(x)+\left(\alpha(t, x)-\int_{0}^{t} \bar{K}_{1}(t-s) \partial_{s} \alpha(s, x) d s\right) \xi \\
& +\overline{\bar{K}}(t) v^{0}(x)+\int_{0}^{t} \overline{\bar{K}}(t-s) f(s, x) d s \quad \text { a.e. }(t, x) \in Q_{T},
\end{aligned}
$$


where

$$
\overline{\bar{K}}(t):=\int_{Y_{2}} \bar{K}(t, y) d y \text { for } t \in(0, T) .
$$

Then, equation (2.15) reduces to

$$
\begin{cases}\partial_{t t}(\bar{u} \cdot \xi)-\operatorname{div}_{x}\left(A_{1}^{*} \nabla_{x} \alpha\right)=f \cdot \xi+\operatorname{div}_{x}\left(\mathbf{A}_{1}^{*} \mathbf{e}_{x}\left(u^{0}\right) \xi\right) & \text { in } Q_{T} \\ \bar{u}(0, x) \cdot \xi=u^{0}(x) \cdot \xi & \text { in } \Omega\end{cases}
$$

Moreover, under the assumptions of Proposition 2.6 we have by (2.22) and (2.23)

$$
\bar{K}_{1}(0)=\left|Y_{2}\right| .
$$

where by (2.24) the function $\alpha$ satisfies the Volterra equation

$$
\begin{aligned}
& \alpha(t, x)-\int_{0}^{t} \bar{K}_{1}(t-s) \partial_{s} \alpha(s, x) d s \\
& =(\bar{u} \cdot \xi)(t, x)-\left(u^{0}(x)+\overline{\bar{K}}(t) v^{0}(x)+\int_{0}^{t} \overline{\bar{K}}(t-s) f(s, x) d s\right) \cdot \xi
\end{aligned}
$$

By virtue of [15, Theorem 16, Chap. 3] there exists a distribution $L \in \mathscr{D}^{\prime}(0, \infty)$ such that the solution $\alpha$ to the above Volterra equation can be expressed with the kernel $L$ as

$$
\begin{aligned}
\alpha(t, x)= & \int_{0}^{t} L(t-s)(\bar{u} \cdot \xi)(s, x) d s \\
& -\int_{0}^{t} L(t-s)\left(u^{0}(x)+\overline{\bar{K}}(s) v^{0}(x)+\int_{0}^{s} \overline{\bar{K}}(s-r) f(r, x) d r\right) \cdot \xi d s .
\end{aligned}
$$

Therefore, noting that the former relation reads as (1.2), equation (2.25) leads us to equation (1.3) together with the stress law (1.4) which can be regarded as a kind of viscoelasticity equation satisfied by the limit displacement $\bar{u} \cdot \xi$ in the direction of the magnetic field with a memory term depending on the initial conditions $u^{0}, v^{0}$ and the force $f$.

\section{Proof of the results}

\subsection{Proof of Theorem 2.1}

Let us start showing some a priori estimate satisfied by the solution $u_{\varepsilon}$ to equation (2.2). For this purpose, we firstly assume that for some fixed $\varepsilon>0$, the functions $f, u^{0}$ and $v^{0}$ satisfy the following regularity conditions:

$$
f \in W^{1, \infty}\left(0, T ; L^{2}(\Omega)\right)^{3}, \quad v^{0} \in H_{0}^{1}(\Omega)^{3}, \quad-\operatorname{Div}\left(A_{\varepsilon}\left(\frac{x}{\varepsilon}\right) \mathbf{e}\left(u^{0}\right)\right)+\frac{1}{\varepsilon} b\left(\frac{x}{\varepsilon}\right) \times v^{0} \in L^{2}(\Omega)^{3} .
$$

Then, the solution $u_{\varepsilon}$ to $(2.2)$ belongs to $W^{2, \infty}\left(0, T ; L^{2}(\Omega)\right)^{3} \cap W^{1, \infty}\left(0, T ; H_{0}^{1}(\Omega)\right)^{3}$, and $\partial_{t} u_{\varepsilon}$ is the unique solution in $W^{1, \infty}\left(0, T ; L^{2}(\Omega)\right)^{3} \cap L^{\infty}\left(0, T ; H_{0}^{1}(\Omega)\right)^{3}$ to equation

$$
\begin{cases}\partial_{t t}^{2}\left(\partial_{t} u_{\varepsilon}\right)-\operatorname{Div}\left(\mathbf{A}_{\varepsilon}\left(\frac{x}{\varepsilon}\right) \mathbf{e}\left(\partial_{t} u_{\varepsilon}\right)\right)+\frac{1}{\varepsilon} b\left(\frac{x}{\varepsilon}\right) \times \partial_{t}\left(\partial_{t} u_{\varepsilon}\right)=\partial_{t} f & \text { in } Q_{T} \\ \partial_{t} u_{\varepsilon}=0 & \text { on }(0, T) \times \partial \Omega \\ \partial_{t} u_{\varepsilon}(0, \cdot)=v^{0}, \quad \partial_{t}\left(\partial_{t} u_{\varepsilon}\right)(0, \cdot)=\operatorname{Div}\left(A_{\varepsilon}\left(\frac{x}{\varepsilon}\right) \mathbf{e}\left(u^{0}\right)\right)-\frac{1}{\varepsilon} b\left(\frac{x}{\varepsilon}\right) \times v^{0}+f(0, \cdot) & \text { in } \Omega .\end{cases}
$$


This allows us to take $\partial_{t} u_{\varepsilon}$ as test function in (2.2). Hence, integrating on $(0, t) \times \Omega$ for each $t \in[0, T]$, we deduce the estimate

$$
\begin{aligned}
& \left\|u_{\varepsilon}\right\|_{W^{1, \infty}\left(0, T ; L^{2}(\Omega)\right)^{3}}+\left\|\mathbf{e}\left(u_{\varepsilon}\right)\right\|_{L^{\infty}\left(0, T ; L^{2}\left(\Omega_{\varepsilon, 1}\right)\right)^{3 \times 3}}+\varepsilon\left\|\mathbf{e}\left(u_{\varepsilon}\right)\right\|_{L^{\infty}\left(0, T ; L^{2}\left(\Omega_{\varepsilon, 2}\right)\right)^{3 \times 3}} \\
& \leq C\left(\|f\|_{L^{2}\left(Q_{T}\right)^{3}}+\left\|u^{0}\right\|_{H_{0}^{1}(\Omega)^{3}}+\left\|v^{0}\right\|_{L^{2}(\Omega)^{3}}\right)
\end{aligned}
$$

where $C$ is a positive constant only depending on $T, \mathbf{A}_{\mathbf{1}}, \mathbf{A}_{\mathbf{2}}$. Now, for functions $f \in L^{2}\left(Q_{T}\right)^{3}$, $u^{0} \in H_{0}^{1}(\Omega)^{3}$ and $v^{0} \in L^{2}(\Omega)^{3}$, consider a sequence $\left(f_{n}\right)_{n \in \mathbb{N}}$ in $W^{1, \infty}\left(0, T ; L^{2}(\Omega)\right)^{3}$ converging strongly to $f$ in $L^{2}\left(Q_{T}\right)^{3}$, a sequence $\left(v_{n}^{0}\right)_{n \in \mathbb{N}}$ in $H_{0}^{1}(\Omega)^{3}$ converging strongly to $v^{0}$ in $L^{2}(\Omega)^{3}$ and a sequence $\left(g_{n}\right)_{n \in \mathbb{N}}$ in $L^{2}(\Omega)^{3}$ with

$$
g_{n} \rightarrow-\operatorname{Div}\left(A_{\varepsilon}\left(\frac{x}{\varepsilon}\right) e\left(u^{0}\right)\right)+\frac{1}{\varepsilon} b\left(\frac{x}{\varepsilon}\right) \times v^{0} \text { strongly in } H^{-1}(\Omega)^{3} .
$$

Then, defining $u_{n}^{0}$ for $n \in \mathbb{N}$, as the unique solution in $H_{0}^{1}(\Omega)^{3}$ to the elasticity equation

$$
\left\{\begin{array}{l}
-\operatorname{Div}\left(A_{\varepsilon}\left(\frac{x}{\varepsilon}\right) e\left(u_{n}^{0}\right)\right)+\frac{1}{\varepsilon} b\left(\frac{x}{\varepsilon}\right) \times v_{n}^{0}=g_{n} \text { in } \Omega \\
u_{n}^{0}=0 \text { on } \partial \Omega
\end{array}\right.
$$

the functions $f_{n}, u_{n}^{0}$ and $v_{n}^{0}$ satisfy conditions (3.1). Also note that for a fixed $\varepsilon>0$, the sequence $\left(u_{n}^{0}\right)_{n \in \mathbb{N}}$ converges strongly in $H_{0}^{1}(\Omega)^{3}$ to $u^{0}$. Hence, estimate (3.2) holds true for the solution $u_{\varepsilon, n}$ to the equation (2.2) with data $f_{n}, u_{n}^{0}$ and $v_{n}^{0}$. Finally, passing to the limit as $n \rightarrow \infty$ in this estimate, we get that estimate (3.2) is still valid for data $f \in L^{2}\left(Q_{T}\right)^{3}$, $u^{0} \in H_{0}^{1}(\Omega)^{3}$ and $v^{0} \in L^{2}(\Omega)^{3}$.

Thanks to estimate (3.2) the two-scale convergence theory of Nguetseng-Allaire [4, 13] provides the existence of functions $u \in W^{1, \infty}\left(0, T ; L^{2}\left(\Omega ; L_{\sharp}^{2}(Y)\right)\right)^{3} \cap L^{\infty}\left(0, T ; L^{2}\left(\Omega ; H_{\sharp}^{1}(Y)\right)\right)^{3}$ and $u_{3} \in L^{\infty}\left(0, T ; L^{2}\left(\Omega ; H_{\sharp}^{1}(Y)\right)\right)^{3}$ such that $u=u(t, x, y)$ is independent of $y$ in $Y_{1}$. Then, defining $u_{1}(t, x):=\chi_{Y_{1}}(y) u(t, x, y)$ for a.e. $(t, x, y) \in(0, T) \times \Omega \times Y_{1}$, the function $u_{1}$ belongs to $L^{\infty}\left(0, T ; H_{0}^{1}(\Omega)\right)^{3}$ and

$$
u_{\varepsilon} \stackrel{2 s}{\rightarrow} u, \quad \partial_{t} u_{\varepsilon} \stackrel{2 s}{\longrightarrow} \partial_{t} u \quad \chi_{\Omega_{\varepsilon, 1}} \mathbf{e}\left(u_{\varepsilon}\right) \stackrel{2 s}{\longrightarrow} \chi_{Y_{1}}\left(\mathbf{e}_{x}\left(u_{1}\right)+\mathbf{e}_{y}\left(u_{3}\right)\right), \quad \chi_{\Omega_{\varepsilon, 2}} \varepsilon \mathbf{e}\left(u_{\varepsilon}\right) \stackrel{2 s}{\longrightarrow} \mathbf{e}_{y}(u) .
$$

Taking $u_{2}=u-u_{1}$, the functions $u_{1}, u_{2}, u_{3}$ satisfy the three first conditions of (2.5) and condition (2.4).

Let us use (2.4) to pass to the limit in (2.2). First, we obtain the initial condition for $u_{1}$, $u_{2}$ at $t=0$. For this purpose we take $\delta>0$ and $\varphi \in C^{0}\left(\Omega ; L_{\sharp}^{2}(Y)\right)^{3}$. We have

$$
\int_{0}^{\delta} \int_{\Omega}\left(u_{\varepsilon}(s, x)-u^{0}(x)\right) \cdot \varphi\left(x, \frac{x}{\varepsilon}\right) d x d s=\int_{0}^{\delta} \int_{0}^{s} \int_{\Omega} \partial_{t} u_{\varepsilon}(r, x) \cdot \varphi\left(x, \frac{x}{\varepsilon}\right) d x d r d s,
$$

which passing to the limit in $\varepsilon$ and using Fubini's theorem yields

$$
\int_{0}^{\delta} \int_{\Omega} \int_{Y}\left(u_{1}+u_{2}-u^{0}\right) \cdot \varphi d y d x d s=\int_{0}^{\delta} \int_{\Omega} \int_{Y}(\delta-r) \partial_{t}\left(u_{1}+u_{2}\right) \cdot \varphi d y d x d r
$$

and thus

$$
\begin{aligned}
& \left|\int_{0}^{\delta} \int_{\Omega} \int_{Y}\left(u_{1}+u_{2}-u^{0}\right) \cdot \varphi d y d x d s\right| \\
& \leq \delta\left(\int_{0}^{\delta} \int_{\Omega} \int_{Y}\left|\partial_{t}\left(u_{1}+u_{2}\right)\right|^{2} d y d x d t\right)^{\frac{1}{2}}\left(\int_{0}^{\delta} \int_{\Omega} \int_{Y}|\varphi|^{2} d y d x d t\right)^{\frac{1}{2}} .
\end{aligned}
$$


Using that $u_{1}+u_{2}$ belongs to $C^{0}\left([0, T] ; L^{2}\left(\Omega ; L_{\sharp}^{2}(Y)\right)\right)^{3}$, we can divide by $\delta$ the former inequality and take the limit as $\delta$ tends to zero, which implies that

$$
u_{1}(0, x)+u_{2}(0, x, y)=u^{0}(x) \text { a.e. }(x, y) \in \Omega \times Y \text {. }
$$

Hence, recalling that $u_{2}$ belongs to $L^{\infty}\left(0, T ; L^{2}\left(\Omega ; H_{0}^{1}\left(Y_{2}\right)\right)\right)^{3}$, we obtain

$$
u_{1}(0, x)=u^{0}(x), \quad u_{2}(0, x, y)=0 \quad \text { a.e. }(x, y) \in \Omega \times Y .
$$

First, we take $\varepsilon \varphi_{2}(t, x, x / \varepsilon)$ with $\varphi_{2} \in C_{c}^{1}\left([0, T) ; H_{0}^{1}\left(Y_{2} ; C^{1}(\bar{\Omega})\right)\right)^{3}$ as test function in $(2.2)$. By virtue of $\left[4\right.$, Remark 1.5] $\varphi_{2}$ is an admissible test function for two-scale convergence. Note that we make no regularity assumption with respect to the variable $y$ in order to preserve the pointwise condition (2.7) for the regularization final step. Then, passing to the limit in (2.2) with the two-scale limits $(2.4)$ we get that

$$
\int_{Q_{T} \times Y_{2}} b \times\left(\partial_{t} u_{1}+\partial_{t} u_{2}\right) \cdot \varphi_{2} d t d x d y=0
$$

or equivalently,

$$
b(y) \times\left(\partial_{t} u_{1}(t, x)+\partial_{t} u_{2}(t, x, y)\right)=0 \text { a.e. }(t, x, y) \in Q_{T} \times Y_{2},
$$

which is the last equality in (2.5).

Now, for

$$
\left\{\begin{array}{c}
\varphi_{1} \in C_{c}^{1}([0, T) \times \Omega)^{3}, \quad \varphi_{2} \in C_{c}^{1}\left([0, T) ; H_{0}^{1}\left(Y_{2} ; C^{1}(\bar{\Omega})\right)\right)^{3}, \quad \varphi_{3} \in C_{c}^{1}\left([0, T) ; H_{\sharp}^{1}\left(Y ; C^{1}(\bar{\Omega})\right)\right)^{3}, \\
\text { with } b(y) \times\left(\varphi_{1}(t, x)+\varphi_{2}(t, x, y)\right)=0 \quad \text { a.e. }(t, x, y) \in Q_{T} \times Y_{2},
\end{array}\right.
$$

we put

$$
\varphi_{\varepsilon}(t, x)=\varphi_{1}(t, x)+\varphi_{2}\left(t, x, \frac{x}{\varepsilon}\right)+\varepsilon \varphi_{3}\left(t, x, \frac{x}{\varepsilon}\right)
$$

as test function in (2.2), and we pass to the limit. The main difficulty comes from the term

$$
\frac{1}{\varepsilon} \int_{Q_{T}}\left(b\left(\frac{x}{\varepsilon}\right) \times \partial_{t} u_{\varepsilon}\right) \cdot\left(\varphi_{1}(t, x)+\varphi_{2}\left(t, x, \frac{x}{\varepsilon}\right)+\varepsilon \varphi_{3}\left(t, x, \frac{x}{\varepsilon}\right)\right) d t d x .
$$

First, using (2.4) and (3.4), we have

$$
\begin{aligned}
& \int_{Q_{T}}\left(b\left(\frac{x}{\varepsilon}\right) \times \partial_{t} u_{\varepsilon}\right) \cdot \varphi_{3}\left(t, x, \frac{x}{\varepsilon}\right) d x=\int_{Q_{T} \times Y}\left(b \times\left(\partial_{t} u_{1}+\partial_{t} u_{2}\right)\right) \cdot \varphi_{3} d t d x d y+o_{\varepsilon}(1) \\
& =\int_{Q_{T} \times Y_{1}}\left(b \times \partial_{t} u_{1}\right) \cdot \varphi_{3} d t d x d y+o_{\varepsilon}(1) .
\end{aligned}
$$

For the remaining term, note that by virtue of Lax Milgram's theorem combined with Korn's inequality, condition (2.3) implies that there exists a unique vector-valued function $U^{i}$ in $H_{\sharp}^{1}\left(Y_{1} ; \mathbb{R}^{3}\right) / \mathbb{R}^{3}$ (i.e., up to an additive constant vector) solution to the Neumann elasticity problem whose variational formulation is

$$
\forall V \in H_{\sharp}^{1}\left(Y_{1} ; \mathbb{R}^{3}\right) / \mathbb{R}^{3}, \quad \int_{Y_{1}} \mathbf{e}\left(U^{i}\right)(y): \mathbf{e}(V)(y) d y=\int_{Y_{1}}\left(b(y) \times e_{i}\right) \cdot V(y) d y,
$$


where $\left(e_{1}, e_{2}, e_{3}\right)$ is the canonical basis of $\mathbb{R}^{3}$. Hence, the symmetric matrix-valued function $G_{i}:=\mathbf{e}\left(U^{i}\right) \in L_{\sharp}^{2}\left(Y_{1} ; \mathbb{R}_{s}^{3 \times 3}\right)$ for $i=1,2,3$, is solution to the equation

$$
\begin{cases}b \times e_{i}=-\operatorname{Div}\left(G^{i}\right) & \text { in } Y_{1} \\ G^{i} \nu=0 & \text { on } \partial Y_{2}\end{cases}
$$

Then, by (3.5) and (2.1) we can write

$$
\begin{aligned}
& \frac{1}{\varepsilon} \int_{Q_{T}}\left(b\left(\frac{x}{\varepsilon}\right) \times \partial_{t} u_{\varepsilon}\right) \cdot\left(\varphi_{1}(t, x)+\varphi_{2}\left(t, x, \frac{x}{\varepsilon}\right)\right) d t d x \\
& =\frac{1}{\varepsilon} \int_{(0, T) \times \Omega_{\varepsilon, 1}}\left(b\left(\frac{x}{\varepsilon}\right) \times \partial_{t} \varphi_{1}\right) \cdot u_{\varepsilon} d t d x+\frac{1}{\varepsilon} \int_{\Omega_{\varepsilon, 1}}\left(b\left(\frac{x}{\varepsilon}\right) \times \varphi_{1}(0, x)\right) \cdot u^{0} d x \\
& =-\sum_{i=1}^{3} \int_{(0, T) \times \Omega_{\varepsilon, 1}} \operatorname{Div}_{x}\left[G^{i}\left(\frac{x}{\varepsilon}\right)\right] \cdot u_{\varepsilon} \partial_{t} \varphi_{1, i} d t d x-\sum_{i=1}^{3} \int_{\Omega_{\varepsilon, 1}} \operatorname{Div}_{x}\left[G^{i}\left(\frac{x}{\varepsilon}\right)\right] \cdot u^{0} \varphi_{1, i}(0, x) d x \\
& =\sum_{i=1}^{3} \int_{(0, T) \times \Omega_{\varepsilon, 1}} G^{i}\left(\frac{x}{\varepsilon}\right): \mathbf{e}\left(u_{\varepsilon}\right) \partial_{t} \varphi_{1, i} d t d x+\sum_{i=1}^{3} \int_{(0, T) \times \Omega_{\varepsilon, 1}} G^{i}\left(\frac{x}{\varepsilon}\right):\left(u_{\varepsilon} \odot \nabla_{x} \partial_{t} \varphi_{1, i}\right) d t d x \\
& +\sum_{i=1}^{3} \int_{\Omega_{\varepsilon, 1}} G^{i}\left(\frac{x}{\varepsilon}\right): \mathbf{e}\left(u^{0}\right) \varphi_{1, i}(0, x) d x+\sum_{i=1}^{3} \int_{\Omega_{\varepsilon, 1}} G^{i}\left(\frac{x}{\varepsilon}\right):\left(u^{0} \odot \nabla_{x} \varphi_{1, i}(0, x)\right) d x \\
& =\sum_{i=1}^{3}\left(\int_{Q_{T} \times Y_{1}} G^{i}:\left(\mathbf{e}_{x}\left(u_{1} \partial_{t} \varphi_{1, i}\right)+\mathbf{e}_{y}\left(u_{3}\right) \partial_{t} \varphi_{1, i}\right) d t d x d y+\int_{\Omega \times Y_{1}} G^{i}: \mathbf{e}_{x}\left(u^{0} \varphi_{1, i}\right) d x d y\right)+o_{\varepsilon}(1),
\end{aligned}
$$

which using the definition (3.6) of $G,(3.3)$ and (2.3) yields

$$
\lim _{\varepsilon \rightarrow 0} \frac{1}{\varepsilon} \int_{Q_{T}}\left(b\left(\frac{x}{\varepsilon}\right) \times \partial_{t} u_{\varepsilon}\right) \cdot\left(\varphi_{1}(t, x)+\varphi_{2}\left(t, x, \frac{x}{\varepsilon}\right)\right) d t d x=-\int_{Q_{T} \times Y_{1}}\left(b \times u_{3}\right) \cdot \partial_{t} \varphi_{1} d t d x d y .
$$

Then, taking into account this equality we have for any functions $\varphi_{1}, \varphi_{2}, \varphi_{3}$ satisfying (3.5),

$$
\begin{aligned}
& -\int_{Q_{T} \times Y}\left(\partial_{t} u_{1}+\partial_{t} u_{2}\right) \cdot\left(\partial_{t} \varphi_{1}+\partial_{t} \varphi_{2}\right) d t d x d y-\int_{\Omega \times Y} v^{0} \cdot\left(\varphi_{1}+\varphi_{2}\right)(0, x, y) d x d y \\
& +\int_{Q_{T} \times Y_{1}} \mathbf{A}_{1}\left(\mathbf{e}_{x}\left(u_{1}\right)+\mathbf{e}_{y}\left(u_{3}\right)\right):\left(\mathbf{e}_{x}\left(\varphi_{1}\right)+\mathbf{e}_{y}\left(\varphi_{3}\right)\right) d t d x d y+\int_{Q_{T} \times Y_{2}} \mathbf{A}_{2} \mathbf{e}_{y}\left(u_{2}\right): \mathbf{e}_{y}\left(\varphi_{2}\right) d t d x d y \\
& +\int_{Q_{T} \times Y_{1}}\left(b \times \partial_{t} u_{1}\right) \cdot \varphi_{3} d t d x d y-\int_{Q_{T} \times Y_{1}}\left(b \times u_{3}\right) \cdot \partial_{t} \varphi_{1} d t d x d y \\
& =\int_{Q_{T} \times Y} f \cdot\left(\varphi_{1}+\varphi_{2}\right) d t d x d y,
\end{aligned}
$$

where $u_{1}, u_{2}$ satisfy $(3.4)$.

Finally, recall that the convolution by a sequence of mollifiers in $C_{c}^{\infty}\left(\mathbb{R} \times \mathbb{R}^{3}\right)$ with respect to the variables $(t, x)$ of a function $\varphi(t, x, y)$ in $L^{1}\left(0, T ; L^{2}\left(\Omega ; H_{\sharp}^{1}(Y)\right)\right)^{3}$ gives a sequence of functions in $C^{1}\left([0, T] ; H_{\sharp}^{1}\left(Y ; C^{1}(\bar{\Omega})\right)\right)^{3}$ (also taking into account the interior cone property of $\Omega$ combined with a partition of unity for the convolution with respect to the variable $x$ ) which strongly converges to $\varphi(t, x, y)$. Hence, by a density argument based on such a regularization the previous equation which holds for regular functions satisfying (3.5) also holds for any functions $\varphi_{1}, \varphi_{2}, \varphi_{3}$ satisfying (2.7), which yields the desired variational problem (2.6). Note that the 
regularization with respect to the variables $(t, x)$ applied to functions $\varphi_{1}, \varphi_{2}, \varphi_{3}$ satisfying (2.7) preserves the pointwise condition of (2.7) in (3.5), since this pointwise condition involves the sole variable $y$ through the vector field $b(y)$.

It remains to prove the quasi-uniqueness of the solutions to problem (2.6). Due to the linearity of (2.6) it is enough to prove that if functions $z_{1}, z_{2}, z_{3}$ satisfying

$$
\left\{\begin{array}{c}
z_{1} \in W^{1,1}\left(0, T ; L^{2}(\Omega)\right)^{3} \cap L^{1}\left(0, T ; H_{0}^{1}(\Omega)\right)^{3}, z_{1}(0, \cdot)=0 \text { in } \Omega, \\
z_{2} \in W^{1,1}\left(0, T ; L^{2}\left(\Omega ; L^{2}\left(Y_{2}\right)\right)\right)^{3} \cap L^{1}\left(0, T ; L^{2}\left(\Omega ; H_{0}^{1}\left(Y_{2}\right)\right)\right)^{3}, z_{2}(0, \cdot, \cdot)=0 \text { in } \Omega \times Y_{2}, \\
z_{3} \in L^{1}\left(0, T ; L^{2}\left(\Omega ; H_{\sharp}^{1}\left(Y_{1}\right)\right)\right)^{3}, \\
b(y) \times\left(z_{1}(t, x)+z_{2}(t, x, y)\right)=0 \text { a.e. }(t, x, y) \in Q_{T} \times Y_{2},
\end{array}\right.
$$

are solutions to problem

$$
\left\{\begin{array}{l}
-\int_{Q_{T} \times Y}\left(\partial_{t} z_{1}+\partial_{t} z_{2}\right) \cdot\left(\partial_{t} \varphi_{1}+\partial_{t} \varphi_{2}\right) d t d x d y \\
+\int_{Q_{T} \times Y_{1}} \mathbf{A}_{1}\left(\mathbf{e}_{x}\left(z_{1}\right)+\mathbf{e}_{y}\left(z_{3}\right)\right):\left(\mathbf{e}_{x}\left(\varphi_{1}\right)+\mathbf{e}_{y}\left(\varphi_{3}\right)\right) d t d x d y+\int_{Q_{T} \times Y_{2}} \mathbf{A}_{2} \mathbf{e}_{y}\left(z_{2}\right): \mathbf{e}_{y}\left(\varphi_{2}\right) d t d x d y \\
+\int_{Q_{T} \times Y_{1}}\left(b \times \partial_{t} z_{1}\right) \cdot \varphi_{3} d t d x d y-\int_{Q_{T} \times Y_{1}}\left(b \times z_{3}\right) \cdot \partial_{t} \varphi_{1} d t d x d y=0,
\end{array}\right.
$$

for any functions $\varphi_{1}, \varphi_{2}, \varphi_{3}$ satisfying

$$
\left\{\begin{array}{c}
\varphi_{1} \in W^{1, \infty}\left(0, T ; L^{2}(\Omega)\right)^{3} \cap L^{\infty}\left(0, T ; H_{0}^{1}(\Omega)\right)^{3}, \varphi_{1}(T, \cdot)=0 \text { in } \Omega, \\
\varphi_{2} \in W^{1, \infty}\left(0, T ; L^{2}\left(\Omega \times Y_{2}\right)\right)^{3} \cap L^{\infty}\left(0, T ; L^{2}\left(\Omega ; H_{0}^{1}\left(Y_{2}\right)\right)\right)^{3}, \varphi_{2}(T, \cdot, \cdot)=0 \text { in } \Omega \times Y_{2}, \\
\varphi_{3} \in L^{\infty}\left(0, T ; L^{2}\left(\Omega ; H_{\sharp}^{1}\left(Y_{1}\right)\right)\right)^{3}, \\
b(y) \times\left(\varphi_{1}(t, x)+\varphi_{2}(t, x, y)\right)=0 \text { a.e. }(t, x, y) \in Q_{T} \times Y_{2},
\end{array}\right.
$$

then we have

$$
z_{1}(t, x)=z_{2}(t, x, y)=0 \quad \text { a.e. }(t, x, y) \in Q_{T} \times Y, \quad \mathbf{e}_{y}\left(z_{3}\right)=0 \quad \text { a.e. }(t, x, y) \in Q_{T} \times Y_{1} .
$$

Indeed, the last equality combined with the periodicity in $y$ shows that

$$
z_{3}(t, x, y)=\lambda(t, x) \text { a.e. }(t, x, y) \in Q_{T} \times Y_{1},
$$

for some $\lambda(t, x) \in \mathbb{R}^{3}$.

To prove this we consider the following dual problem. For any $g \in L^{2}\left(Q_{T} \times Y\right)^{3}$, let functions $\psi_{1}, \psi_{2}, \psi_{3}$ satisfying

$$
\left\{\begin{array}{c}
\psi_{1} \in W^{1, \infty}\left(0, T ; L^{2}(\Omega)\right)^{3} \cap L^{\infty}\left(0, T ; H_{0}^{1}(\Omega)\right)^{3}, \psi_{1}(T, \cdot)=0, \\
\psi_{2} \in W^{1, \infty}\left(0, T ; L^{2}\left(\Omega ; L^{2}\left(Y_{2}\right)\right)\right)^{3} \cap L^{\infty}\left(0, T ; L^{2}\left(\Omega ; H_{0}^{1}\left(Y_{2}\right)\right)\right)^{3}, \psi_{2}(T, \cdot, \cdot)=0, \\
\psi_{3} \in L^{\infty}\left(0, T ; L^{2}\left(\Omega ; H_{\sharp}^{1}\left(Y_{1}\right)\right)\right)^{3}, \\
b(y) \times\left(\psi_{1}(t, x)+\psi_{2}(t, x, y)\right)=0 \text { a.e. }(t, x, y) \in Q_{T} \times Y_{2},
\end{array}\right.
$$


be solutions to the dual problem to $(2.6)$

$$
\begin{aligned}
& -\int_{Q_{T} \times Y}\left(\partial_{t} \psi_{1}+\partial_{t} \psi_{2}\right) \cdot\left(\partial_{t} \varphi_{1}+\partial_{t} \varphi_{2}\right) d t d x d y \\
& +\int_{Q_{T} \times Y_{1}} \mathbf{A}_{1}\left(\mathbf{e}_{x}\left(\psi_{1}\right)+\mathbf{e}_{y}\left(\psi_{3}\right)\right):\left(\mathbf{e}_{x}\left(\varphi_{1}\right)+\mathbf{e}_{y}\left(\varphi_{3}\right)\right) d t d x d y+\int_{Q_{T} \times Y_{2}} \mathbf{A}_{2} \mathbf{e}_{y}\left(\psi_{2}\right): \mathbf{e}_{y}\left(\varphi_{2}\right) d t d x d y \\
& +\int_{Q_{T} \times Y_{1}}\left(b \times \partial_{t} \psi_{1}\right) \cdot \varphi_{3} d t d x d y-\int_{Q_{T} \times Y_{1}}\left(b \times \psi_{3}\right) \cdot \partial_{t} \varphi_{1} d t d x d y \\
& =\int_{Q_{T} \times Y} g \cdot\left(\varphi_{1}+\varphi_{2}\right) d t d x d y
\end{aligned}
$$

for any functions $\varphi_{1}, \varphi_{2}, \varphi_{3}$ satisfying

$$
\left\{\begin{array}{c}
\varphi_{1} \in W^{1,1}\left(0, T ; L^{2}(\Omega)\right)^{3} \cap L^{1}\left(0, T ; H_{0}^{1}(\Omega)\right)^{3}, \varphi_{1}(0, \cdot)=0 \text { in } \Omega, \\
\varphi_{2} \in W^{1,1}\left(0, T ; L^{2}\left(\Omega \times Y_{2}\right)\right)^{3} \cap L^{1}\left(0, T ; L^{2}\left(\Omega ; H_{0}^{1}\left(Y_{2}\right)\right)\right)^{3}, \varphi_{2}(0, \cdot, \cdot)=0 \text { in } \Omega \times Y_{2}, \\
\varphi_{3} \in L^{1}\left(0, T ; L^{2}\left(\Omega ; H_{\sharp}^{1}\left(Y_{1}\right)\right)\right)^{3}, \\
b(y) \times\left(\varphi_{1}(t, x)+\varphi_{2}(t, x, y)\right)=0 \text { a.e. }(t, x, y) \in Q_{T} \times Y_{2} .
\end{array}\right.
$$

Using the change of variables $s=T-t$, the existence of solutions $\psi_{1}, \psi_{2}, \psi_{3}$ to problem (3.12) follows from the existence of solutions $z_{1}, z_{2}, z_{3}$ to problem (3.8) which is given by the two-scale convergence.

Then, taking $\psi_{1}, \psi_{2}, \psi_{3}$ as test functions in (3.8) and taking $z_{1}, z_{2}, z_{3}$ as test functions in (3.12), we get that

$$
\int_{Q_{T} \times Y} g \cdot\left(z_{1}+z_{2}\right) d t d x d y=0, \quad \forall g \in L^{2}\left(Q_{T} \times Y\right)^{3},
$$

which implies that

$$
z_{1}(t, x)+z_{2}(t, x, y)=0 \text { a.e. }(t, x, y) \in Q_{T} \times Y .
$$

This combined with $z_{2} \in L^{1}\left(0, T ; L^{2}\left(\Omega ; H_{0}^{1}\left(Y_{2}\right)\right)\right)^{3}$ yields the two first equalities of $(3.10)$. Moreover, taking $\varphi_{1}=\varphi_{2}=0$ in (3.8) we get that

$$
\int_{Q_{T} \times Y_{1}} \mathbf{A}_{1} \mathbf{e}_{y}\left(z_{3}\right): \mathbf{e}_{y}\left(\varphi_{3}\right) d t d x d y=0, \quad \forall \varphi_{3} \in L^{\infty}\left(0, T ; L^{2}\left(\Omega ; H_{\sharp}^{1}\left(Y_{1}\right)\right)\right)^{3},
$$

which implies the last equality of (3.10).

This concludes the proof of Theorem 2.1.

\subsection{Proof of Theorem 2.2}

Let us solve problem (2.6). First, we take $\varphi_{1}=\varphi_{3}=0$, then we get

$$
\begin{aligned}
& -\int_{Q_{T} \times Y_{2}}\left(\partial_{t} u_{1}+\partial_{t} u_{2}\right) \cdot \partial_{t} \varphi_{2} d t d x d y-\int_{\Omega \times Y} v^{0} \cdot \varphi_{2}(0, x, y) d x d y \\
& +\int_{Q_{T} \times Y_{2}} \mathbf{A}_{2} \mathbf{e}_{y}\left(u_{2}\right): \mathbf{e}_{y}\left(\varphi_{2}\right) d t d x d y=\int_{Q_{T} \times Y} f \cdot \varphi_{2} d t d x d y
\end{aligned}
$$

where $\varphi_{2}$ is such that $b \times \varphi_{2}=0$. 
Under assumption (2.8) define the spaces

$$
H_{2}:=\left\{\psi \in L^{2}\left(Y_{2}\right)^{3}: \psi \times b=0\right\}, \quad V_{2}:=H_{2} \cap H_{0}^{1}\left(Y_{2}\right)^{3} .
$$

Then, $\varphi_{2} \in W^{1,1}\left(0, T ; L^{2}\left(\Omega ; H_{2}\right)\right) \cap L^{1}\left(0, T ; L^{2}\left(\Omega ; V_{2}\right)\right)$. Moreover, observe that condition (3.4) can be written as

$$
\partial_{t} u_{1}+\partial_{t} u_{2} \in H_{2} \text { a.e. }(t, x) \in[0, T) \times \Omega,
$$

which taking into account (3.3) implies that

$$
u_{1}+u_{2}-u^{0} \in V_{2}
$$

Then, define the functions

$$
\left\{\begin{array}{l}
v_{1}(t, x, y):=\frac{b(y) \otimes b(y)}{|b|^{2}} u_{1}(t, x), \quad v_{2}(t, x, y):=\frac{b(y) \otimes b(y)}{|b(y)|^{2}} u_{2}(t, x, y) \\
\text { a.e. }(t, x, y) \in Q_{T} \times Y_{2},
\end{array}\right.
$$

i.e. $v_{1}$ and $v_{2}$ are the orthogonal projections of $u_{1}$ and $u_{2}$ on the direction of $b$, and note that by $(3.15) v_{2}$ still belongs to $L^{\infty}\left(0, T ; L^{2}\left(\Omega ; H_{0}^{1}\left(Y_{2}\right)\right)\right)^{3}$. This allows us to write $(2.6)$ as

$$
\begin{aligned}
& -\int_{0}^{T} \int_{Y_{2}}\left(\partial_{t} v_{1}+\partial_{t} v_{2}\right) \cdot \partial_{t} \varphi_{2} d t d y-\int_{Y_{2}} v^{0} \cdot \varphi_{2}(0, y) d y \\
& +\int_{0}^{T} \int_{Y_{2}} \mathbf{A}_{2} \mathbf{e}_{y}\left(v_{2}\right): \mathbf{e}_{y}\left(\varphi_{2}\right) d t d y=\int_{0}^{T} \int_{Y_{2}} f \cdot \varphi_{2} d t d y \\
& \text { a.e. } x \in \Omega, \forall \varphi_{2} \in W^{1,1}\left(0, T ; H_{2}\right) \cap L^{1}\left(0, T ; V_{2}\right) .
\end{aligned}
$$

Choosing $\varphi_{2}$ with $\varphi_{2}(0, \cdot)=0$, this shows that $v_{1}, v_{2}$ satisfy

$$
\frac{d^{2}}{d t^{2}} \int_{Y_{2}}\left(v_{1}+v_{2}\right) \cdot \psi_{2} d y+\int_{Y_{2}} \mathbf{A}_{2} \mathbf{e}_{y}\left(v_{2}\right): \mathbf{e}_{y}\left(\psi_{2}\right) d y=\int_{Y_{2}} f \cdot \psi_{2} d y, \quad \forall \psi_{2} \in V_{2},
$$

which combined with (3.17) yields the initial condition

$$
\left(\partial_{t} v_{1}+\partial_{t} v_{2}\right)(0, x, y)=\frac{b(y) \otimes b(y)}{|b(y)|^{2}} v^{0}(x) \text { a.e. }(t, x, y) \in \Omega \times Y_{2}
$$

Now, let $h_{j}, j \geq 1$, be an orthonormal basis in $H_{2}$ of eigenvectors in $V_{2}$ associated with the eigenvalues $\mu_{j}^{2}$ of the spectral problem

$$
\left\{\begin{array}{l}
h_{j} \in V_{2}, \quad \text { with } \bar{h}_{j}:=\int_{Y_{2}} h_{j} d y \\
\int_{Y_{2}} \mathbf{A}_{2} \mathbf{e}_{y}\left(h_{j}\right): \mathbf{e}_{y}\left(\psi_{2}\right) d y=\mu_{j}^{2} \int_{Y_{2}} h_{j} \cdot \psi_{2} d y, \quad \forall \psi_{2} \in V_{2}
\end{array}\right.
$$

Such an orthonormal basis does exist, since $H_{2}$ and $V_{2}$ are closed subspaces of $L^{2}\left(Y_{2}\right)^{3}$ and $H_{0}^{1}\left(Y_{2}\right)^{3}$ respectively and $V_{2}$ is dense in $H_{2}$ (recalling that by (2.8) $b$ is a non-vanishing function in $H^{1}\left(Y_{2}\right)^{3}$, the subspace of $V_{2}$ composed of functions $\alpha b$ with $\alpha \in C_{c}^{1}\left(Y_{2}\right)$, is dense in $\left.H_{2}\right)$. Hence, the embedding of $V_{2}$ into $H_{2}$ remains compact so that the discrete spectral decomposition of a self-adjoint compact operator applies. 
Since $v_{2} \in V_{2}$, we have

$$
v_{2}(t, x, y)=\sum_{j=1}^{\infty} \phi_{j}(t, x) h_{j}(y) \text { a.e. }(t, x, y) \in Q_{T} \times Y_{2} .
$$

Putting this series in (3.18) with the test function $\psi_{2}=h_{i}, i \geq 1$, adding the term $\mu_{i}^{2} v_{1} \cdot \bar{h}_{i}$ in both sides and taking into account the initial conditions (3.3) and (3.19), we get that

$$
\left\{\begin{array}{l}
\frac{\partial^{2}}{\partial t^{2}}\left(v_{1} \cdot \bar{h}_{i}+\phi_{i}\right)+\mu_{i}^{2}\left(v_{1} \cdot \bar{h}_{i}+\phi_{i}\right)=\left(f+\mu_{i}^{2} v_{1}\right) \cdot \bar{h}_{i} \text { in }(0, T) \text { a.e. } x \in \Omega \\
\left(v_{1} \cdot \bar{h}_{i}+\phi_{i}\right)(0, x)=u^{0}(x) \cdot \bar{h}_{i}, \partial_{t}\left(v_{1} \cdot \bar{h}_{i}+\phi_{i}\right)(0, x)=v^{0}(x) \cdot \bar{h}_{i} .
\end{array}\right.
$$

which leads us to

$$
\begin{aligned}
\left(v_{1} \cdot \bar{h}_{i}+\phi_{i}\right)(t, x) & =\int_{0}^{t} \frac{\sin \left(\mu_{i}(t-s)\right)}{\mu_{i}}\left(f(s, x)+\mu_{i}^{2} v_{1}(s, x)\right) \cdot \bar{h}_{i} d s \\
& +\cos \left(\mu_{i} t\right) u^{0}(x) \cdot \bar{h}_{i}+\frac{\sin \left(\mu_{i} t\right)}{\mu_{i}} v^{0}(x) \cdot \bar{h}_{i} .
\end{aligned}
$$

Integrating by parts and again using (3.3) yields

$$
\begin{aligned}
\phi_{i}(t, x) & =\frac{\sin \left(\mu_{i} t\right)}{\mu_{i}} \bar{h}_{i} \cdot v^{0}(x)+\int_{0}^{t} \frac{\sin \left(\mu_{i}(t-s)\right)}{\mu_{i}} \bar{h}_{i} \cdot f(s, x) d s \\
& -\int_{0}^{t} \cos \left(\mu_{i}(t-s)\right) \bar{h}_{i} \cdot \partial_{s} v_{1}(s, x) d s
\end{aligned}
$$

Hence, by summing with respect to $i$ we get that

$$
\begin{aligned}
v_{2}(t, x, y) & =\sum_{i=1}^{\infty} \frac{\sin \left(\mu_{i} t\right)}{\mu_{i}}\left(h_{i}(y) \otimes \bar{h}_{i}\right) v^{0}(x)+\sum_{i=1}^{\infty} \int_{0}^{t} \frac{\sin \left(\mu_{i}(t-s)\right)}{\mu_{i}}\left(h_{i}(y) \otimes \bar{h}_{i}\right) f(s, x) d s \\
& -\sum_{i=1}^{\infty} \int_{0}^{t} \cos \left(\mu_{i}(t-s)\right)\left(h_{i}(y) \otimes \bar{h}_{i}\right) \partial_{s} v_{1}(s, x) d s
\end{aligned}
$$

Finally, defining the kernel

$$
\bar{K}(t, y):=\sum_{i=1}^{\infty} \frac{\sin \left(\mu_{i} t\right)}{\mu_{i}} h_{i}(y) \otimes \bar{h}_{i}, \quad \text { for }(t, y) \in(0, T) \times Y_{2},
$$

we obtain

$$
\left\{\begin{array}{l}
v_{2}(t, x, y)=\bar{K}(t, y) v^{0}(x)+\int_{0}^{t} \bar{K}(t-s, y) f(s, x) d s-\int_{0}^{t} \partial_{t} \bar{K}(t-s, y) \partial_{s} u_{1}(s, x) d s \\
\text { a.e. }(t, x, y) \in Q_{T} \times Y_{2} .
\end{array}\right.
$$

We have replaced in (3.23) the function $v_{1}$ by the function $u_{1}$ which are connected by (3.16), since that for a.e. $(t, y) \in(0, T) \times Y_{2}$ the range of $\bar{K}(t, y)$ is contained in the space spanned by $b(y)$. On the other hand, note that using the series expansion (3.22) and

$$
\sum_{i=1}^{\infty}\left|\bar{h}_{i}\right|^{2}<\infty
$$


we can check that

$$
\bar{K} \in L^{\infty}\left(0, T ; V_{2}\right)^{3} \cap W^{1, \infty}\left(0, T ; H_{2}\right)^{3} \cap W^{2, \infty}\left(0, T ; V_{2}^{\prime}\right)^{3} .
$$

Moreover, since $V_{2} \subset H_{0}^{1}\left(Y_{2}\right)^{3}$ and the range of $\bar{K}$ is contained in the space spanned by $b$, the kernel satisfies the regularity (2.11). Formula (3.23) also gives an expression of $u_{2}$, since by (3.15) and (3.16) we have

$$
u_{2}=v_{2}+\left(I-\frac{b \otimes b}{|b|^{2}}\right)\left(u^{0}-u_{1}\right)
$$

which gives the desired expression (2.14).

Let us now compute the function $u_{3}$ in problem (2.6). We choose $\varphi_{1}=\varphi_{2}=0$. We get

$$
\int_{Q_{T} \times Y_{1}} \mathbf{A}_{1}\left(\mathbf{e}_{x}\left(u_{1}\right)+\mathbf{e}_{y}\left(u_{3}\right)\right): \mathbf{e}_{y}\left(\varphi_{3}\right) d t d x d y+\int_{Q_{T} \times Y_{1}}\left(b \times \partial_{t} u_{1}\right) \cdot \varphi_{3} d t d x d y=0 .
$$

Let $w_{j k}$ and $\vartheta_{j}, 1 \leq j, k \leq 3$, be the vector-valued functions defined by the cell problems

$$
\left\{\begin{array}{l}
w_{j k} \in H_{\sharp}^{1}\left(Y_{1}\right)^{3} \\
\int_{Y_{1}} \mathbf{A}_{1}\left(E_{j k}+\mathbf{e}_{y}\left(w_{j k}\right)\right): \mathbf{e}_{y}(\psi) d y=0, \quad \forall \psi \in H_{\sharp}^{1}\left(Y_{1}\right)^{3},
\end{array}\right.
$$

where $\left(E_{j k}\right)_{1 \leq j, k \leq 3}$ is the canonical basis in $\mathbb{R}_{s}^{3 \times 3}$,

$$
\left\{\begin{array}{l}
\vartheta_{j} \in H_{\sharp}^{1}\left(Y_{1}\right)^{3} \\
\int_{Y_{1}} \mathbf{A}_{1} \mathbf{e}_{y}\left(\vartheta_{j}\right): \mathbf{e}_{y}(\psi) d y+\int_{Y_{1}}\left(b \times e_{j}\right) \cdot \psi d y=0, \quad \forall \psi \in H_{\sharp}^{1}\left(Y_{1}\right)^{3} .
\end{array}\right.
$$

Then, defining $\mathbf{W}(y): \mathbb{R}^{3 \times 3} \rightarrow \mathbb{R}^{3}$ and $V(y) \in \mathbb{R}^{3 \times 3}$ by

$$
\mathbf{W}(y) M:=\sum_{j, k=1}^{3} m_{j k} w_{j k}(y), \quad V(y) \eta:=\sum_{j=1}^{3} \eta_{j} \vartheta_{j}(y), \quad \forall M \in \mathbb{R}^{3 \times 3}, \forall \eta \in \mathbb{R}^{3},
$$

the function $u_{3}$ is given by

$$
u_{3}(t, x, y)=\mathbf{W}(y) \mathbf{e}_{x}\left(u_{1}\right)(t, x)+V(y) \partial_{t} u_{1}(t, x) \text { a.e. }(t, x, y) \in Q_{T} \times Y_{1}
$$

\section{Case where magnetic field has one direction on the boundary of the inclusion}

Assume that $b_{\mid \partial Y_{2}}$ has a fixed direction $\xi$ with $|\xi|=1$. Then, by (2.5) and (2.8) there exists a scalar function $\alpha \in W^{1, \infty}\left(0, T ; L^{2}(\Omega)\right) \times L^{\infty}\left(0, T ; H_{0}^{1}(\Omega)\right)$ such that $(2.13)$ holds. For any $\beta \in W^{2, \infty}\left(0, T ; L^{2}(\Omega)\right)^{3} \times W^{1, \infty}\left(0, T ; H_{0}^{1}(\Omega)\right)^{3}$ with $\beta(0, x)=\beta(T, x)=0$, we define

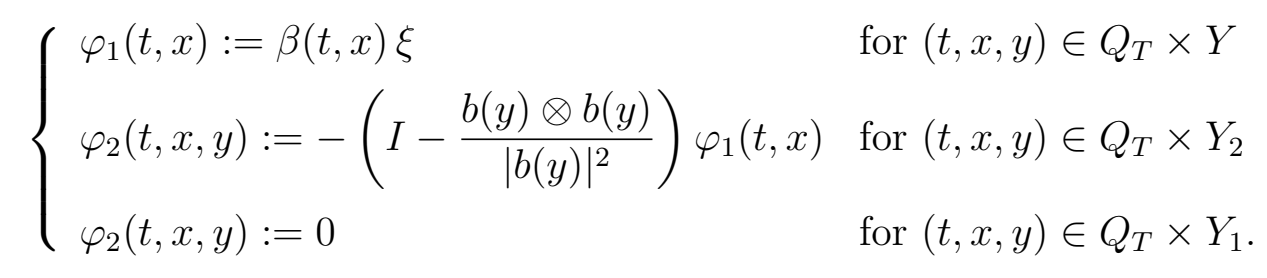


Taking $\varphi_{3}=0$ in $(2.6)$ we have

$$
\begin{aligned}
& -\int_{Q_{T} \times Y}\left(\partial_{t} u_{1}+\partial_{t} u_{2}\right) \cdot\left(\partial_{t} \varphi_{1}+\partial_{t} \varphi_{2}\right) d t d x d y \\
& +\int_{Q_{T} \times Y_{1}} \mathbf{A}_{1}\left(\mathbf{e}_{x}\left(u_{1}\right)+\mathbf{e}_{y}\left(u_{3}\right)\right): \mathbf{e}_{x}\left(\varphi_{1}\right) d t d x d y+\int_{Q_{T} \times Y_{2}} \mathbf{A}_{2} \mathbf{e}_{y}\left(u_{2}\right): \mathbf{e}_{y}\left(\varphi_{2}\right) d t d x d y \\
& -\int_{Q_{T} \times Y_{1}}\left(b \times u_{3}\right) \cdot \partial_{t} \varphi_{1} d t d x d y=\int_{Q_{T} \times Y} f \cdot\left(\varphi_{1}+\varphi_{2}\right) d t d x d y .
\end{aligned}
$$

Since by (2.13) $u_{1}=u^{0}+\alpha \xi$ and by (3.15)

$$
u_{1}+u_{2}-u^{0}=\frac{b \otimes b}{|b|^{2}}\left(u_{1}+u_{2}-u^{0}\right)
$$

by the definitions (3.16) of $v_{2}$ and (2.16) of $\hat{b}$ we also have

$$
u_{1}(t, x)+u_{2}(t, x, y)=u^{0}(x)+v_{2}(t, x, y)+\alpha(t, x) \hat{b}(y) \text { a.e. }(t, x, y) \in Q_{T} \times Y_{2} .
$$

Then, using the expressions (3.28) of $u_{3}$ and (3.29) of $\varphi_{1}, \varphi_{2}$, and (2.17) we get that

$$
\begin{aligned}
& -\int_{Q_{T}}\left(\left|Y_{1}\right|+\int_{Y_{2}}|\hat{b}|^{2} d y\right) \partial_{t} \alpha \partial_{t} \beta d t d x+\int_{Q_{T}}\left(\int_{Y_{2}} v_{2} \cdot \hat{b} d y\right) \partial_{t t}^{2} \beta d t d x \\
& +\int_{Q_{T}} \mathbf{A}_{1}^{*} \mathbf{e}_{x}\left(u^{0}+\alpha \xi\right): \mathbf{e}_{x}(\beta \xi) d t d x+\int_{Q_{T}} \partial_{t} \alpha V_{1}^{*}: \mathbf{e}_{x}(\beta \xi) d t d x \\
& +\int_{Q_{T} \times Y_{2}} \mathbf{A}_{2} \mathbf{e}_{y}\left(\alpha \hat{b}+v_{2}\right): \mathbf{e}_{y}(\beta \hat{b}) d t d x d y \\
& -\int_{Q_{T}}\left(\mathbf{w}^{*} \mathbf{e}_{x}(\alpha \xi)+m^{*} \partial_{t} \alpha\right) \partial_{t} \beta d t d x=\int_{Q_{T}} f \cdot\left(\left|Y_{1}\right| \cdot \xi+\int_{Y_{2}} \hat{b} d y\right) \beta d t d x,
\end{aligned}
$$

where $\mathbf{A}_{1}^{*} \in \mathscr{L}\left(\mathbb{R}_{s}^{3 \times 3}\right), V_{1}^{*} \in \mathbb{R}_{s}^{3 \times 3}, \mathbf{w}^{*}: \mathbb{R}^{3 \times 3} \rightarrow \mathbb{R}, m^{*}$ are the homogenized quantities defined by

$$
\begin{cases}\mathbf{A}_{1}^{*} E_{j k}:=\int_{Y_{1}} \mathbf{A}_{1}\left(E_{j k}+e_{y}\left(w_{j k}\right)\right) d y, & 1 \leq j, k \leq 3, \\ V_{1}^{*}:=\sum_{j=1}^{3} \xi_{j} \int_{Y_{1}} \mathbf{e}_{y}\left(\vartheta_{j}\right) d y . & 1 \leq j, k \leq 3 \\ \mathbf{w}^{*} E_{j k}:=\xi \cdot \int_{Y_{1}} b \times w_{j k} d y & \\ m^{*}:=\xi \cdot \int_{Y_{1}} b \times(V \xi) d y=\sum_{j, k=1}^{3}\left(\int_{Y_{1}} \mathbf{A}_{1} \mathbf{e}_{y}\left(\vartheta_{j}\right): \mathbf{e}_{y}\left(\vartheta_{k}\right) d y\right) \xi_{j} \xi_{k} . & \end{cases}
$$


This can also be written as

$$
\begin{aligned}
& -\int_{Q_{T}}\left(\left|Y_{1}\right|+\int_{Y_{2}}|\hat{b}|^{2} d y\right) \partial_{t} \alpha \partial_{t} \beta d t d x+\int_{Q_{T}}\left(\int_{Y_{2}} v_{2} \cdot \hat{b} d y\right) \partial_{t t}^{2} \beta d t d x \\
& +\int_{Q_{T}} \mathbf{A}_{1}^{*}\left(\mathbf{e}_{x}\left(u^{0}\right)+\nabla_{x} \alpha \odot \xi\right):\left(\nabla_{x} \beta \odot \xi\right) d t d x+\int_{Q_{T}} \partial_{t} \alpha V_{1}^{*}:\left(\nabla_{x} \beta \odot \xi\right) d t d x \\
& +\int_{Q_{T}}\left(\int_{Y_{2}} \mathbf{A}_{2} \mathbf{e}_{y}(\hat{b}): \mathbf{e}_{y}(\hat{b}) d y\right) \alpha \beta d t d x+\int_{Q_{T}}\left(\int_{Y_{2}} \mathbf{A}_{2} \mathbf{e}_{y}\left(v_{2}\right): \mathbf{e}_{y}(\hat{b}) d y\right) \beta d t d x \\
& -\int_{Q_{T}}\left(\mathbf{w}^{*}\left(\nabla_{x} \alpha \odot \xi\right)+m^{*} \partial_{t} \alpha\right) \partial_{t} \beta d t d x=\int_{Q_{T}} f \cdot\left(\left|Y_{1}\right| \xi+\int_{Y_{2}} \hat{b} d y\right) \beta d t d x .
\end{aligned}
$$

Defining

$$
\left\{\begin{array}{l}
M^{*}:=\left|Y_{1}\right|+m^{*}+\int_{Y_{2}}|\hat{b}|^{2} d y \\
c^{*}:=\int_{Y_{2}} \mathbf{A}_{2} \mathbf{e}_{y}(\hat{b}): \mathbf{e}_{y}(\hat{b}) d y \\
\lambda^{*} \cdot \zeta:=\mathbf{w}^{*}(\xi \odot \zeta)-V_{1}^{*} \xi \cdot \zeta, \quad \text { for } \zeta \in \mathbb{R}^{3} \\
\mu^{*}:=\left|Y_{1}\right| \xi+\int_{Y_{2}} \hat{b} d y \\
A_{1}^{*} \zeta:=\mathbf{A}_{1}^{*}(\zeta \odot \xi) \xi, \quad \text { for } \zeta \in \mathbb{R}^{3},
\end{array}\right.
$$

and using the representation (3.23) of $v_{2}$ the previous variational formulation leads us to the following distributional equation

$$
\begin{aligned}
& \partial_{t t}\left(M^{*} \alpha\right)-\partial_{t t}\left[\int_{0}^{t}\left(\int_{Y_{2}} \partial_{t} \bar{K}(t-s, y):(\hat{b}(y) \odot \xi) d y\right) \partial_{s} \alpha(s, x) d s\right] \\
& +\lambda^{*} \cdot \nabla_{x}\left(\partial_{t} \alpha\right)-\operatorname{div}_{x}\left(A_{1}^{*} \nabla_{x} \alpha\right)+c^{*} \alpha-\int_{Y_{2}} \mathbf{A}_{2} \mathbf{e}_{y}\left(\int_{0}^{t} \partial_{s} \alpha(s, x) \partial_{t} \bar{K}(t-s, y) \xi d s\right): \mathbf{e}_{y}(\hat{b}) d y \\
& =-\partial_{t t}\left[\int_{Y_{2}} \bar{K}(t, y):\left(\hat{b}(y) \otimes v^{0}(x)\right) d y\right]-\int_{Y_{2}} \mathbf{A}_{2} \mathbf{e}_{y}\left(\bar{K}(t, y) v^{0}(x)\right): \mathbf{e}_{y}(\hat{b}) d y \\
& +\mu^{*} \cdot f-\partial_{t t}\left[\int_{0}^{t}\left(\int_{Y_{2}} \bar{K}(t-s, y) f(s, x) d y\right) \cdot \hat{b}(y) d s\right] \\
& -\int_{Y_{2}} \mathbf{A}_{2} \mathbf{e}_{y}\left(\int_{0}^{t} \bar{K}(t-s, y) f(s, x) d s\right): \mathbf{e}_{y}(\hat{b}) d y+\operatorname{div}_{x}\left(\mathbf{A}_{1}^{*} \mathbf{e}_{x}\left(u^{0}\right) \xi\right),
\end{aligned}
$$

which by the definition $(2.11)$ of the kernel $\bar{K}$ also can be written as

$$
\begin{aligned}
& \partial_{t t}\left[M^{*} \alpha-\int_{0}^{t}\left(\int_{Y_{2}} \partial_{t} \bar{K}(t-s, y):(\xi \odot \xi) d y\right) \partial_{s} \alpha(s, x) d s\right] \\
& +\lambda^{*} \cdot \nabla_{x}\left(\partial_{t} \alpha\right)-\operatorname{div}_{x}\left(A_{1}^{*} \nabla_{x} \alpha\right)+c^{*} \alpha-\int_{Y_{2}} \mathbf{A}_{2} \mathbf{e}_{y}\left(\int_{0}^{t} \partial_{s} \alpha(s, x) \partial_{t} \bar{K}(t-s, y) \xi d s\right): \mathbf{e}_{y}(\hat{b}) d y \\
& =-\partial_{t t}\left[\int_{Y_{2}} \bar{K}(t, y):\left(\xi \otimes v^{0}(x)\right) d y\right]-\int_{Y_{2}} \mathbf{A}_{2} \mathbf{e}_{y}\left(\bar{K}(t, y) v^{0}(x)\right): \mathbf{e}_{y}(\hat{b}) d y+\mu^{*} \cdot f \\
& -\partial_{t t}\left[\int_{Y_{2}}\left(\int_{0}^{t} \bar{K}(t-s, y) f(s, x) d s\right) \cdot \xi d y\right]-\int_{Y_{2}} \mathbf{A}_{2} \mathbf{e}_{y}\left(\int_{0}^{t} \bar{K}(t-s, y) f(s, x) d s\right): \mathbf{e}_{y}(\hat{b}) d y \\
& +\operatorname{div}_{x}\left(\mathbf{A}_{1}^{*} \mathbf{e}_{x}\left(u^{0}\right) \xi\right) .
\end{aligned}
$$


This provides the homogenized equation (2.15) satisfied by $u_{1}(t, x)=u^{0}(x)+\alpha(t, x) \xi$.

\section{Case where magnetic field has two directions on the boundary of the inclusion}

Finally, assume that $b_{\mid \partial Y_{2}}$ has at least two independent directions. Due to the regularity of $b$ equality (3.4) yields

$$
b(y) \times \partial_{t} u_{1}(t, x)=0 \text { a.e. }(t, x, y) \in Q_{T} \times \partial Y_{2},
$$

which clearly implies (2.10). Moreover, the proof of formula (2.12) is quite similar to the proof of (2.14) in the previous case.

It remains to prove the uniqueness of the solution $\alpha$ to equation (2.15). To this end, consider a solution $\omega \in W^{1, \infty}\left(0, T ; L^{2}(\Omega)\right) \cap L^{\infty}\left(0, T ; H_{0}^{1}(\Omega)\right)$ of equation $(2.15)$ with nul right-hand side, i.e.

$$
\left\{\begin{array}{l}
\partial_{t t}\left[M^{*} \omega-\int_{0}^{t} \bar{K}_{1}(t-s) \partial_{s} \omega(s, x) d s\right]+\lambda^{*} \cdot \nabla_{x}\left(\partial_{t} \omega\right)-\operatorname{div}_{x}\left(A_{1}^{*} \nabla_{x} \omega\right) \\
+c^{*} \omega-\int_{Y_{2}} \mathbf{A}_{2} \mathbf{e}_{y}\left(\int_{0}^{t} \partial_{s} \omega(s, x) \partial_{t} \bar{K}(t-s, y) \xi d s\right): \mathbf{e}_{y}(\hat{b}) d y=0 \text { in } Q_{T} \\
\omega(0, \cdot)=0 \text { in } \Omega .
\end{array}\right.
$$

Then, going back up the former calculations, the functions $z_{1}, z_{2}, z_{3}$ given respectively from the definitions (2.13), (2.14), (3.28) of $u_{1}, u_{2}, u_{3}$, by

$$
\left\{\begin{array}{l}
z_{1}(t, x)=\omega(t, x) \xi \\
z_{2}(t, x, y)=-\int_{0}^{t} \partial_{t} \bar{K}(t-s, y) \partial_{s} z_{1}(s, x) d s-\left(I-\frac{b(y) \otimes b(y)}{|b(y)|^{2}}\right) \omega(t, x) \xi \\
z_{3}(t, x, y)=\mathbf{W}(y) \mathbf{e}_{x}\left(z_{1}\right)(t, x)+V(y) \partial_{t} z_{1}(t, x), \\
\text { a.e. }(t, x, y) \in Q_{T} \times Y_{2},
\end{array}\right.
$$

are solutions to the homogeneous variational problem (3.8) whose solutions are given by (3.10). Hence, we obtain that $\omega(t, x)=0$ a.e. $(t, x) \in Q_{T}$.

The proof of Theorem 2.2 is now complete.

\subsection{Proof of Proposition 2.6}

By (3.20) and the series expansion (3.22) of $\bar{K}$, the scalar function $\bar{k}:=\bar{K}:(\xi \otimes \xi)$ is solution to the equation

$$
\begin{cases}\partial_{t t}^{2} \bar{k}-\operatorname{div}\left(A_{2} \nabla \bar{k}\right)=0 & \text { in }(0, T) \times Y_{2} \\ \bar{k}(t, \cdot)=0 & \text { on }(0, T) \times \partial Y_{2} \\ \bar{k}(0, \cdot)=0, \partial_{t} \bar{k}(0, \cdot)=1 & \text { in } Y_{2}\end{cases}
$$

where $A_{2}$ is the positive definite symmetric matrix of $\mathbb{R}^{3 \times 3}$ defined by

$$
A_{2} \zeta:=\mathbf{A}_{2}(\zeta \odot \xi) \xi, \text { for } \zeta \in \mathbb{R}^{3} .
$$

By a regularization procedure we may put identical unity as test function in the equation (3.33), which after an integration by parts leads us to the formula

$$
\partial_{t t}^{2}\left(\int_{Y_{2}} \bar{k}(t, y) d y\right)=\int_{\partial Y_{2}} A_{2} \nabla \bar{k} \cdot n d \sigma(y) .
$$


Then, using the estimate of [10, Theorem 4.1]:

$$
A_{2} \nabla \bar{k} \cdot n \in L^{\infty}\left(0, T ; L^{2}\left(\partial Y_{2}\right)\right),
$$

we get that

$$
\partial_{t t}^{2}\left(\int_{Y_{2}} \bar{k}(t, y) d y\right) \in L^{\infty}(0, T) .
$$

This combined with definition (2.17) implies that

$$
\bar{K}_{1}(t)=\int_{Y_{2}} \partial_{t} \bar{k}(t, y) d y \in W^{1, \infty}(0, T) .
$$

Acknowledgment. The authors wish to thank the unknown referee for his/her very careful reading and relevant comments which have improved the presentation of the paper.

\section{References}

[1] Z. Abdessamad, I. Kostin, Ilya; G. Panasenko \& V.P. Smyshlyaev: "Memory effect in homogenization of a viscoelastic Kelvin-Voigt model with time-dependent coefficients", Math. Models Methods Appl. Sci., 19 (9) (2009), 1603-1630.

[2] D.I. Bardzokas, M.L. Filshtinsky \& L.A. Filshtinsky: Mathematical Methods in Electro-Magneto-Elasticity, Lecture Notes in Appl. and Compt. Mech. 32, Springer-Verlag Berlin 2007, pp. 530.

[3] A. Ávila; G. Griso, B. Miara, E. Rohan: "Multiscale modeling of elastic waves: theoretical justification and numerical simulation of band gaps", Multiscale Model. Simul., 7 (1) (2008), 1-21.

[4] G. Allaire: "Homogenization and two-scale convergence", SIAM J. Math. Anal., 23 (6) (1992), 1482-1518.

[5] M. Briane \& J. Casado-Díaz: "Increase of mass and nonlocal effects in the homogenization of magneto-elastodynamics problems", arXiv:1806.10998, 2018.

[6] J. Casado-Díaz, J. Couce-Calvo, F. Maestre \& J.D. Martín-Gómez: "Homogenization and correctors for the wave equation with periodic coefficients", Math. Mod. Meth. Appl. Sci., 24 (2014), 1343-1388.

[7] F. Colombini \& S. Spagnolo: "On the convergence of solutions of hyperbolic equations", Comm. Partial Differential Equations, 3 (1) (1978), 77-103.

[8] G.A. Francfort \& F. Murat: "Oscillations and energy densities in the wave equation", Comm. Partial Differential Equations, 17 (1992), 1785-1865.

[9] I.V. Kamotski \& V.P. Smyshlyaev: "Two-scale homogenization for a general class of high contrast PDE systems with periodic coefficients", Applicable Analysis, 98 (2019), 64-90. 
[10] J.-L. Lions: Contrôlabilité exacte, perturbations et stabilisation de systèmes distribués, Tome 1 (French) [Exact controllability, perturbations and stabilization of distributed systems, Vol. 1], Recherches en Mathématiques Appliquées [Research in Applied Mathematics], 8, Masson, Paris, 1988, 541 pp.

[11] J.-L. Lions \& E. MAGenes: Non-homogeneous boundary value problems and applications. Vol. I, Translated from the French by P. Kenneth, Die Grundlehren der mathematischen Wissenschaften, Band 181, Springer-Verlag, New York-Heidelberg, 1972, 373 pp.

[12] G.W. Milton \& J. Willis: "On modifications to Newton's second law and linear continuum elastodynamics", Proc. R. Soc. Lond. Ser. A, Math. Phys. Eng. Sci., 463 (2079) (2007), 855-880.

[13] G. NGuetseng: "A general convergence result for a functional related to the theory of homogenization", SIAM J. Math. Anal. , 20 (3) (1989), 608-623.

[14] E. SÁnchez-Palencia: Nonhomogeneous media and vibration theory, Lecture Notes in Physics 127, Springer-Verlag, Berlin-New York, 1980, 398 pp.

[15] L. Schwartz: Mathematics for the physical sciences, Hermann, Paris, Addison-Wesley Publishing Co., 1966, 358 pp.

[16] V.P. Smyshlyaev: "Propagation and localization of elastic waves in highly anisotropic periodic composites via two-scale homogenization", Mech. Mater., 41 (2009), 434-447.

[17] L. TARTAR: "Homogénéisation en hydrodynamique", in Singular Perturbation and Boundary Layer Theory, Lecture Notes in Mathematics, 597, Springer, Berlin-Heidelberg 1977, 474-481.

[18] V.V. Zhikov \& S.E. Pastukhova: "On gaps in the spectrum of the operator of elasticity theory on a high contrast periodic structure", J. Math. Sci. (N.Y.), 188 (3) (2013), 227-240. 